\title{
Autophagy and Its Impact on Neurodegenerative Diseases: New Roles for TDP-43 and C9orf72
}

\author{
Mauricio Budini ${ }^{1 *}$, Emanuele Buratti ${ }^{2}$, Eugenia Morselli ${ }^{3}$ and Alfredo Criollo ${ }^{1,4 *}$ \\ ${ }^{1}$ Dentistry Faculty, Institute in Dentistry Sciences, University of Chile, Santiago, Chile, ${ }^{2}$ International Centre for Genetic \\ Engineering and Biotechnology, Trieste, Italy, ${ }^{3}$ Department of Physiology, Faculty of Biological Sciences, Pontificia \\ Universidad Católica de Chile, Santiago, Chile, ${ }^{4}$ Advanced Center for Chronic Diseases, Santiago, Chile
}

OPEN ACCESS

Edited by:

Angelo Poletti,

Università degli Studi di Milano, Italy

Reviewed by:

Alessandro Vercelli,

University of Turin, Italy

Cristina Cereda,

Fondazione Istituto Neurologico Nazionale Casimiro Mondino (IRCCS),

Italy

*Correspondence:

Mauricio Budini mbudini@u.uchile.cl

Alfredo Criollo

alcriollo@u.uchile.c

Received: 01 February 2017 Accepted: 15 May 2017 Published: 30 May 2017

Citation:

Budini M, Buratti E, Morselli E and Criollo A (2017) Autophagy and lts

Impact on Neurodegenerative Diseases: New Roles for TDP-43 and

C9orf72.

Front. Mol. Neurosci. 10:170 doi: 10.3389/fnmol.2017.00170
Autophagy is a catabolic mechanism where intracellular material is degraded by vesicular structures called autophagolysosomes. Autophagy is necessary to maintain the normal function of the central nervous system (CNS), avoiding the accumulation of misfolded and aggregated proteins. Consistently, impaired autophagy has been associated with the pathogenesis of various neurodegenerative diseases. The proteins TAR DNA-binding protein-43 (TDP-43), which regulates RNA processing at different levels, and chromosome 9 open reading frame 72 (C9orf72), probably involved in membrane trafficking, are crucial in the development of neurodegenerative diseases such as Amyotrophic lateral sclerosis (ALS) and Frontotemporal Lobar Degeneration (FTLD). Additionally, recent studies have identified a role for these proteins in the control of autophagy. In this manuscript, we review what is known regarding the autophagic mechanism and discuss the involvement of TDP-43 and C9orf72 in autophagy and their impact on neurodegenerative diseases.

\section{Keywords: autophagy, neurodegenerative diseases, TDP-43, C9orf72, ALS, FTLD}

\section{INTRODUCTION}

The population is aging worldwide. Indeed, between 2015 and 2030, the number of people in the world aged 60 years or over is projected to grow by 56 per cent, from 901 million to 1.4 billion; by 2050, the global population of older persons is projected to more than double in size, reaching nearly 2.1 billion $^{1}$ As people age, the risk of developing a neurodegenerative disease rises dramatically (Beghi et al., 2006; Reeve et al., 2014); therefore, the study of the molecular bases of neurological disorders has become an urgent issue (Johnson, 2015).

Neurodegenerative diseases such as Alzheimer's, Parkinson's, Prion, Huntington's, and Motor neuron diseases (MND), as well as Spinal muscular atrophy, Spinocerebellar ataxia (SCA), Frontotemporal Lobar Degeneration (FTLD), and Amyotrophic lateral sclerosis (ALS) cause progressive damage in the central and peripheral nervous system, reducing the quality of life of affected patients (do Carmo Costa and Paulson, 2013). At a biochemical level, various molecular mechanisms underlie the development of neurodegenerative diseases; however, they all lead to chronic loss of function of neurons or nerves (Ciechanover and Kwon, 2015). A characteristic feature of many of these disorders is the accumulation of irregular protein structures in the neurons, which stimulate the formation of inclusion bodies and insoluble deposits (Atkin and Paulson, 2014; Ciechanover and Kwon, 2015).These features are generally associated with

\footnotetext{
${ }^{1}$ http://www.un.org/en/development/desa/population/publications/pdf/ageing/WPA2015_Report.pdf
} 
an inefficient protein quality control system in the neurons, which prevents the elimination of dysfunctional proteins and promotes their accumulation inside the cells (Nijholt et al., 2011).

To date, an effective treatment for these pathologies has not been discovered; only palliative approaches to alleviate patient symptoms are currently used (Lokk and Delbari, 2012; do Carmo Costa and Paulson, 2013). Thus, it is critical to identify novel molecular targets to develop new therapies for their treatment (do Carmo Costa and Paulson, 2013).

The TAR-DNA binding protein 43, TDP-43, and the chromosome 9 open reading frame 72, C9orf72, are closely associated with the development of ALS, a progressive neurodegenerative disease that affects nerve cells in the brain and the spinal cord, and Frontotemporal Lobar Degeneration (FTLD), a form of dementia characterized by a progressive decline in behavior and/or language (Baralle et al., 2013; Cruts et al., 2013; Rohrer et al., 2015). TDP-43 is a nuclear factor involved in molecular pathways that control the fate of cellular RNAs, and, in contrast to healthy neurons, it is found in an aggregated form in neurons of ALS and FTLD patients (Arai et al., 2006; Neumann et al., 2006). The potentially toxic role of TDP-43 aggregates is poorly understood; however, it has been proposed that they might act as "sink" for newly synthetized, functional TDP-43 protein, leading to TDP-43 loss-of-function in the cell (Budini et al., 2014, 2015). Thus, these insoluble aggregates of TDP-43 probably impair the normal processing of specific RNA targets, promoting neuronal cell death and, therefore, neurodegeneration.

To date, the physiological functions C9orf72 have not been completely understood; however, recent studies suggest that C9orf72 can be involved in membrane trafficking and in autophagy (Sellier et al., 2016; Sullivan et al., 2016). For example, C9orf72 generates nuclear RNA granules that abnormally sequester key factors required for cellular homeostasis in both ALS and FTLD patients (Cruts et al., 2013).

Among the different cellular pathways that could be involved in the onset of ALS and FTLD autophagy has recently received considerable attention (Menzies et al., 2015). Together with the proteasome, autophagy has been identified as the main pathway that promotes protein degradation and, when altered, it impairs cell homeostasis and physiology (Nijholt et al., 2011). In this regard, in vivo and in vitro studies support the hypothesis that autophagy is responsible for the degradation of TDP-43 (Scotter et al., 2014). In addition, recent observations suggest that TDP-43 is not just a passive substrate for autophagy; rather, it seems actively involved in its initiation (Bose et al., 2011). Similarly, recent evidences indicate that C9orf72 appears to be a key autophagic regulator, functioning through protein-protein interactions (Webster et al., 2016).

Here, we will discuss evidence of the correlation of TDP43 and C9orf72 with the autophagic pathway. This manuscript will help clarify how the "cross-regulation" between TDP-43 and C9orf72 impacts autophagy and, therefore, the onset and the progression of neurodegenerative diseases such as ALS and FTLD.

\section{AUTOPHAGY}

\section{An Overview of the Autophagy Process}

Autophagy is a regulated auto-degradative intracellular process highly conserved across many species, from yeast to mammals. Macroautophagy, hereafter referred to as "autophagy," begins with the formation of a primitive membrane structure called the "omegasome," which arises from intracellular membranous domains. The omegasome sequesters cytosolic targets (Lamb et al., 2013; Mizumura et al., 2014), which can include aggregates or insoluble proteins, damaged and/or oxidized organelles such as mitochondria (mitophagy), endoplasmic reticulum (ERphagy), peroxisomes (pexophagy), lipid droplets (lipophagy), and ferritin (ferritinophagy) (Iwata et al., 2006; Narendra et al., 2008; Lamb et al., 2013), and becomes known as an autophagosome. The autophagosome, in turn, fuses with a lysosome to form an "autophagolysosome," where the cargo is degraded by hydrolytic lysosomal enzymes in either a bulk or selective manner (Lamb et al., 2013; Mizumura et al., 2014). This autophagic degradation results in the production of available metabolites that support key metabolic cellular requirements (Bento et al., 2016) (Figure 1). Nutritional stress (starvation) represents the most common condition to induce autophagy; however, other stress stimuli such as ER stress, hypoxia, mitochondrial damage, free radicals, and genotoxic stress can also induce it (Bento et al., 2016). Importantly, stressinduced autophagy and basal autophagy are crucial to maintain cellular homeostasis in vitro and in vivo (Fernandez and LopezOtin, 2015). Indeed, unbalanced autophagy, either increased or reduced, contributes to the progression of various human diseases, including neurodegenerative diseases, muscle disorders, cancer, metabolic dysfunctions, cardiovascular, and infectious diseases (Fernandez and Lopez-Otin, 2015).

How can autophagy be involved in the regulation of such a variety of human illnesses and disorders? As previously mentioned, autophagy functions in all cells of the body to remove toxic and damaged material. Thus, a decrement to autophagy promotes the accumulation of material that is normally removed from the cell and adversely affects cell survival.

During autophagy, the process of cargo selection is generally non-selective; however, specific autophagy receptors have been identified that mediate the clearance of specific cargoes (Bento et al., 2016). One of the most studied autophagy receptors is the protein p62/SQSTM1, which is both a selective autophagy substrate and a cargo receptor for autophagic degradation of polyubiquitinated proteins (Katsuragi et al., 2015; Bento et al., 2016). p62/SQSTM1 contains a light chain 3 (LC3)interacting region (LIR), which targets the LC3 anchored in the omegasome membrane; cargoes are thus directed to the nascent autophagosome for clearance (Birgisdottir et al., 2013). Other LIR-containing autophagy receptors are the proteins Bcell lymphoma 2 (Bcl2) Interacting Protein 3 (BNIP3) and NIX, two homologous $\mathrm{Bcl} 2$ homology 3 ( $\mathrm{BH} 3$ )-only proteins, which localize in the outer mitochondrial membrane and mediate mitophagy (autophagic clearance of mitochondria) (Kanki, 2010; Hanna et al., 2012). Both BNIP3 and NIX contain a LIR motif 


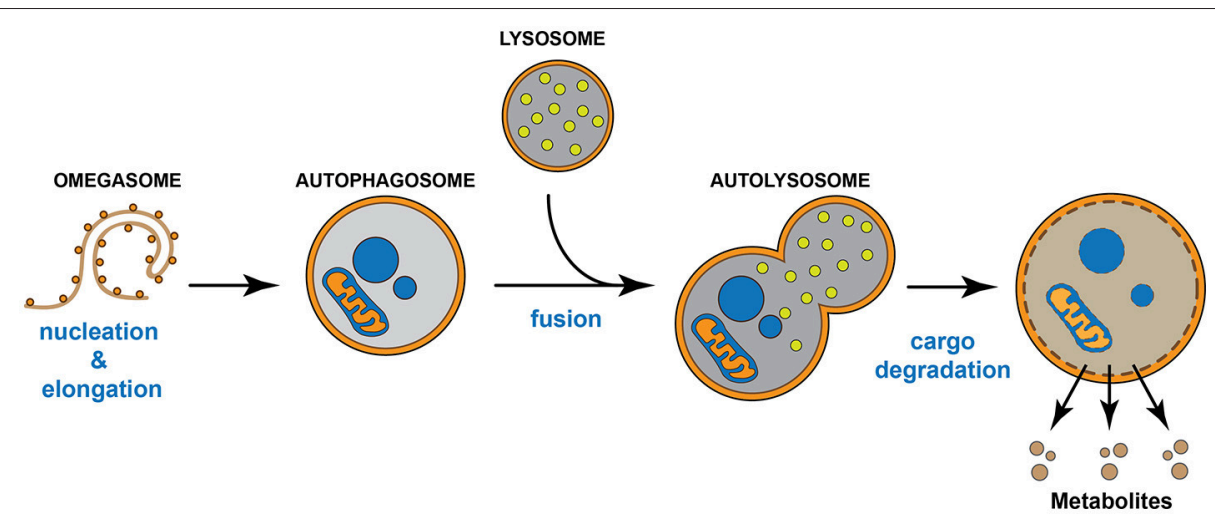

FIGURE 1 | Autophagic pathway. The omegasome expands its membrane to form a double membrane organelle called autophagosome, which sequesters intracellular material such as proteins and organelles. Then, the autophagosome fuses with the lysosome to form the autolysosome. The lysosome supplies hydrolytic enzymes, which are activated following the autophagosome-lysosome fusion, promoting the degradation of the autolysosome cargo. The digestion of the cargo generates new metabolites that turn back to the cytosol and that will be re-used by the cell.

in the amino terminus that faces the cytosol, leading to LIRmediated mitophagy by interaction of the LIR motif with LC3 during autophagosome formation (Kanki, 2010; Hanna et al., 2012).

As mentioned above, the degradation of the autophagic cargo is dependent on lysosomal enzymes. Impaired fusion of the autophagosome with the lysosome, or inhibition of the acidification of the lysosomal compartment prevents the degradation of the autophagosome luminal content and promotes autophagosome accumulation, thus inhibiting "autophagic flux" (Klionsky et al., 2016). The term "autophagic flux" is defined as the difference between the rate of autophagosome formation and cargo clearance by lysosomes (Klionsky et al., 2016). A blockage in the autophagic flux can be observed in different human diseases, and is quite common in neurodegenerative diseases, where an abnormal accumulation of autophagosomes caused by inefficient fusion with lysosomes occurs (Ravikumar et al., 2005). The mechanisms responsible for impairing autophagic flux in neurodegenerative disease vary, and represent an active subject of investigation.

\section{The Autophagy Machinery}

The site where autophagosome assembly occurs in the cell remains controversial, and different locations and organelles have been proposed as the site of assembly and source of the membranes composing the autophagic vacuoles. The ER (Hayashi-Nishino et al., 2009; Yla-Anttila et al., 2009), mitochondria (Hailey et al., 2010), the plasma membrane (Ravikumar et al., 2010; Moreau and Rubinsztein, 2012), the Golgi apparatus (Geng et al., 2010; Bodemann et al., 2011) and mitochondria-associated membranes (MAMs) (Hamasaki et al., 2013; Patergnani et al., 2015) have all been described as subcellular compartments from which the phagophore and the autophagosome might arise. More than 35 autophagyrelated (atg) genes are involved in the process of autophagic vacuole formation (Bento et al., 2016). The primitive phagophore becomes an autophagosome thanks to the activity of the class III phosphoinositide 3-kinase (PI3KC3), which is a large macromolecular complex formed by a catalytic sub-unit the vacuolar protein sorting 34 (VPS34), a regulatory sub-unit VPS15 (p150), ATG6 (also known as Beclin 1 in mammals) and ATG14L (Russell et al., 2014). The PI3KC3 also binds, through Beclin 1, the activating molecule in Beclin 1-related autophagy 1 protein (AMBRA1), which stimulates the activation complex when autophagy is induced (Di Bartolomeo et al., 2010). The proautophagy role of Beclin 1 in the PI3KC3 can be also regulated by $\mathrm{Bcl}-2$ or $\mathrm{Bcl}-\mathrm{XL}$ located at the $\mathrm{ER}$, which down-regulates autophagy by binding Beclin 1, specifically through the Bcl-2 homology type 3 (BH3) domain (Maiuri et al., 2007a,b).

The increased activity of $\mathrm{PI} 3 \mathrm{KC} 3$ generates phosphatidylinositol 3-phosphate (PtdIns3P) in membrane microdomains where proteins with the PtdIns3P-binding (FYVE) and the conserved Phox-homology (PX) domains are recruited to form a protein elongation platform at the omegasome (Simonsen et al., 2004; Zhong et al., 2009). One of these proteins with FYVE domains is the double FYVE domaincontaining protein 1 (DFCP1), which is highly accumulated in a PtdIns3P-dependent manner in conditions of starvation-induced autophagy (Axe et al., 2008). Other proteins whose recruitment is mediated by PtdIns3P are the WD-repeat proteins interacting with phosphoinositides, WIPI-49, which is four member protein family: WIPI1, WIPI2, WIPI3, and WIPI4 (Proikas-Cezanne et al., 2004).

The elongation and expansion of the phagophore requires the concerted actions of two ubiquitin-like conjugation systems (Polson et al., 2010). The first ubiquitin-like conjugation system is formed by ATG12-ATG5-ATG16L. ATG16L1, which is directly bound through WIPI2 to the membrane micro domains, allows the expansion of the phagophore (Itakura and Mizushima, 2010; Walczak and Martens, 2013). Specifically, the activity of this ubiquitin-like conjugation system begins with the conjugation of ATG12 to the lysine residue of ATG5 by the sequential reactions of the E1-like enzyme ATG7, and the E2-like enzyme ATG10. The resulting ATG12-ATG5 conjugate interacts non-covalently 
with ATG16L, and they oligomeryze, forming a large multimeric complex (Hamasaki et al., 2013). In the second ubiquitin-like system, the cytosolic forms of ATG8 (microtubule-associated protein 1 light chain 3 (MAP-LC3), GABARAP and GATE16) are cleaved at their C-terminus by the cysteine protease ATG4B and then conjugated at the amino group with the lipid phosphatidylethanolamine (PE) by ATG7 and by the E2like enzyme ATG3 (Tanida et al., 2004; Katahira et al., 2008). LC3 is the most characterized ATG8 family member and its lipidation with PE generates LC3-II (Otomo et al., 2013). LC3-II is specifically bound to the autophagic vesicle and is widely used as an autophagic marker (Klionsky et al., 2016). Once the autophagosome is formed, the ATG12-ATG5-ATG16L complex associated to the convex surface of the autophagosomal membrane dissociates and is released into the cytoplasm to be reused for the biogenesis of new autophagy vesicles (Lamb et al., 2013).

Once the autophagosomes are fused with the lysosomes, hydrolytic lysosomal enzymes are activated, and they degrade both the luminal content of the autophagosome and its inner membrane. This process of degradation allows the formation of new metabolites that recycle back to the cytoplasm for being reused (Lamb et al., 2013). Autophagosomes fuse with lysosomes by the action of several soluble $\mathrm{N}$-ethylmaleimide-sensitive fusion (NSF) attachment protein receptors (SNARE). These include the vesicle-associated membrane protein (VAMP)8, syntaxin-7, syntaxin-8, and t-SNAREs homolog 1B (Vti1B) (Moreau et al., 2013). Impairment in lysosome-autophagosome fusion induced by dysfunctions in SNARESs proteins decreases the size of the autophagic vesicles and inhibits their maturation into autophagolysosomes. This blockage in the fusion process leads to lysosomal storage disorders, which are characterized by an abnormal accumulation of substrates in the lysosomes (Fraldi et al., 2010; Moreau et al., 2013).

In the regulation of the autophagic process, the most characterized protein regulators are the mechanistic target of rapamycin, mTOR, and the AMP-activated protein kinase (AMPK), which are considered cellular nutritional sensors able to modulate different signaling pathways (Kim et al., 2011; Meijer et al., 2015; Chantranupong and Sabatini, 2016). mTOR is a constitutive Ser/Thr protein kinase sensitive to nutritional and energetic changes, and comprises two large protein complexes, the mTOR complex 1 (mTORC1) and mTOR complex 2 (mTORC2) (Russell et al., 2014). mTORC1 is formed by mTOR, Regulatory-associated protein of mTOR (RAPTOR), mTOR associated protein, LST8 Homolog (mLST8), DEP domain containing mTOR-interacting protein (DEPTOR) and proline-rich Akt substrate of $40 \mathrm{kDa}$ (PRAS40). In basal conditions the kinase activity of mTOR renders unc-51-like kinase 1 protein (ULK1, ortholog of Atg1 in humans) inactive by phosphorylation of Ser757, Ser758 and Ser638 (Kim et al., 2011; Meijer et al., 2015). In these conditions, the ULK complex formed by ULK1, ATG13 and FAK family kinase-interacting protein of $200 \mathrm{kDa}$ (FIP200) remains inactive and autophagy is inhibited (Hara et al., 2008; Wong et al., 2013). In conditions of nutrient deprivation, a low ATP/AMP ratio activates AMPactivated protein kinase (AMPK), which up-regulates the Ser/Thr kinase activity of ULK by phosphorylation on Ser317, Ser777, and Ser555 (Wong et al., 2013). Once activated ULK is released from $\mathrm{mTORC} 1$ and relocates to phagophore assembly sites where it increases the activity of PI3KC3, which, as previously mentioned, generates PtdIns3P in the membrane microdomains (Axe et al., 2008). AMBRA can also regulate the relocalization of PI3KC3 to the autophagosome nucleation sites (Di Bartolomeo et al., 2010). Once autophagy is induced, ULK1 phosphorylates AMBRA, which releases PI3KC3 from the microtubule-associated dynein motor complex, initiating the process of autophagosome elongation (Di Bartolomeo et al., 2010) (Figure 2).

On the other hand, AMPK up-regulates autophagy through phosphorylation of tuberous sclerosis complex 2 (TSC2) at Thr1227, which, together with TSC1, forms the TSC complex (Inoki et al., 2003; Orlova and Crino, 2010). Active TSC2 induces autophagy by suppression of the RAS homolog enriched in brain (Rheb) protein, which is required to maintain mTOR constitutive activity (Orlova and Crino, 2010). AMPK can also directly phosphorylate the mTORC1 subunit RAPTOR, which inhibits mTORC1, thereby inducing autophagy (Gwinn et al., 2008) (Figure 2).

The protein kinase $\mathrm{B}(\mathrm{PKB}$, also known as $\mathrm{AKT})$ is another negative regulator of autophagy (Heras-Sandoval et al., 2014). When nutrients and growth factors are provided, tyrosine kinase receptors are activated by autophosphorylation and stimulate the small GTPase Ras and class I Phosphatidylinositol 3 kinase (PtdIns3K) (Russell et al., 2014). Active class I PtdIns3K increases the levels of $\operatorname{PtdIns(3)P}$ in the membrane, promoting the recruitment and the subsequent activation of AKT (Russell et al., 2014). AKT phosphorylates TSC2 on multiple sites, leading to the disruption of the TSC1-TSC2 complex and promoting mTORC1 activation, thus inhibiting autophagosome formation (Inoki et al., 2002; Potter et al., 2002).

\section{AUTOPHAGY DYSREGULATION IN NEURODEGENERATIVE DISEASES}

Autophagy dysfunction has been identified as a characteristic of the vast majority of neurodegenerative diseases. Components of the autophagic machinery, involved in the different stages of autophagosome formation up to autophagosome-lysosome fusion, can be altered in these illnesses, inhibiting autophagy at various stages along the pathway. This generates different pathological situations, generally characterized by the deposition of aggregate prone proteins in neurons.

Consistently, neuron-specific deletion of essential autophagy genes (Atg5 and Atg7) inhibits autophagy and promotes a neurodegenerative phenotype characterized by axonal degeneration and accumulation of aggregate-prone proteins in the cytosol of neurons without altering proteasome function (Hara et al., 2006; Komatsu et al., 2006). These studies indicate that basal autophagy is required to prevent the accumulation of damaged proteins and toxic material, which, if allowed to accumulate, leads to neurodegeneration. Autophagy is particularly relevant in neurons, which are post-mitotic cells. 




FIGURE 2 | The autophagic machinery. The energetic status of the cell is sensed by the proteins mTOR and AMPK. When nutrients are high mTOR, which, together with Raptor, Deptor, mLST8, and PRAS 40 forms the mTORC1 complex, inhibits the ULK1 kinase activity phosphorylating its Ser638 and Ser757. ULK1, ATG13, and FIP200 form the ULK complex. Upon nutrient deprivation, AMPK is activated and induces autophagy phosphorylating different target proteins. Unlike mTOR, AMPK activates ULK1 by phosphorylation on Ser317 and Ser777, leading to the activation of the PI3KC3 complex, which is formed by AMBRA, PI3KC3, Beclin 1 and ATG14L. AMPK can also induce autophagy by direct suppression of the mTOR pathway phosphorylating Raptor (Ser722 and Ser792) or TSC2 (Thr1227). Active PI3KC3 increases the production of phosphatidylinositol 3-phosphate (Ptdlns3P) in specific membrane micro domains of the plasma membrane, the ER, mitochondria and/or MAMs. Accumulation of Ptdlns3P recruits FYVE domains proteins such as WIPIs and DFCP1. The elongation and expansion of the phagophore membrane are mediated by ATG proteins through two ubiquitination-like systems: the complex ATG12-ATG5-ATG16L and the conjugate ATG8 (LC3)-phosphatidylethanolamine (PE) (also called LC3-II). Finally, while the ATG12-ATG5-ATG16L conjugate is disassembled from the autophagosomal membrane, LC3-II remains enclosed to the inner and the external membrane of the vacuole. Other proteins such as SNARES and RABs are also required for the trafficking of membranes and vacuole elongation. 
Without the aid of cell division, neurons require efficient autophagy to remove the waste material that would otherwise accumulate in the cytosol. Additionally, neuronal autophagy has been shown to be essential not only for proper neuronal function, but also for prevention of inflammation in glial cells, as well as proper oligodendrocyte development and preservation of the myelination process (Lee, 2012).

As indicated in the previous section, the autophagic process includes multiple steps, each of which can be altered in the different types of neurodegenerative diseases.

\section{Dysfunctional Formation of the Isolation Membrane}

In some mouse models of Alzheimer's disease (AD) the gene encoding for Beclin 1 shows decreased mRNA levels in brain tissue, suggesting reduced autophagosome formation and increased development of AD (Pickford et al., 2008). Consistently, crossing mice that overexpress the human amyloid precursor protein, with those carrying a heterozygous deletion of Beclin 1, which per se leads to neurodegeneration, aggravates the AD phenotype (Pickford et al., 2008). Beclin 1 activity is also affected in Huntington disease (HD). Indeed, the mutant Huntington gene, which is critical for the onset of HD, recruits Beclin 1, inhibits its activity and, therefore, autophagosome formation (Shibata et al., 2006). Also, mTOR appears to be sequestered in cell and mouse models of $\mathrm{HD}$, as well as in human brains of HD patients, indicating that the initiation of the autophagic process has been inhibited (Ravikumar et al., 2004).

Additionally, the first step of the autophagic process is compromised in some cases of Parkinson's disease (PD), where omegasomes formation is inhibited due to the mislocalization of the protein ATG9, preventing the formation of new autophagosomes. This reduces the clearance of the protein $\alpha$ synuclein, which accumulates in intra-neuronal inclusions called Lewy bodies, a feature of the brain tissue of PD patients (Winslow et al., 2010).

\section{Cargo Sequestration}

Inefficient recognition of aggregate proteins during the autophagic process has been proposed as a cause of the development of neurodegenerative diseases (Sarkar et al., 2009). As previously described, recognition of certain posttranslational modifications by molecules that bind both cargo and components of the autophagic machinery drives the selectivity of the autophagic cargo (Sarkar et al., 2009). For instance, the protein p62/SQSTM1 is specifically recognized and degraded within autophagosomes (Bjorkoy et al., 2005). Mutations in p62/SQSTM1 have been found in ALS patients, which, as will be better explained in the next sections, leads to p62/SQSTM1 accumulation in inclusion bodies, a typical feature of the disease (Fecto et al., 2011). Also, some forms of HD are characterized by alterations in the process of cargo sequestration. Indeed, due to mutations in the Huntington protein, cellular and mouse models of HD show an inefficient engulfment of cytosolic components in the lumen of autophagosomes, which, even though they form normally, remain empty, thereby promoting the accumulation of the waste material in the cytoplasm of neurons (Martinez-Vicente et al., 2010).

\section{Autophagosome-Lysosome Fusion}

The next step of the autophagic process is the fusion of autophagosomes with lysosomes or endosomes (to later fuse with lysosomes). Autophagosomes move along microtubules in the direction of the perinuclear microtubule-organizing center of the neuron, which is enriched in lysosomes (Lee et al., 2011). Microtubule-based vesicle transport is necessary for efficient autophagy and, when microtubules are disassembled, autophagosomes accumulate in axonal terminals where they cannot be degraded (Hollenbeck, 1993). Mutations that affect Dynein, a protein that promotes the movement on microtubules, impairs autophagosome-lysosome fusion and thus autophagic clearance of aggregate-prone proteins, causing $\mathrm{HD}$ in fly and mouse models (Ravikumar et al., 2005). Similarly, the dynein motor complex is mutated in the axonal Charcot-Marie-Tooth hereditary neuropathy type 2 (CMT2) and loses its function in ALS, promoting autophagosome accumulation (Ferrucci et al., 2011; Sasaki, 2011). Another protein that is necessary for autophagosome-lysosome fusion is RAB7, a late endosome protein, which influences lysosome positioning and thus the autophagosome-lysosome fusion process (Dodson et al., 2012; Uusi-Rauva et al., 2012). This protein has been found mutated in some forms of CMT2 (Spinosa et al., 2008). Additionally, mutations in the protein charged multivesicular body protein 2B (CHMP2B) affecting the activity of the endosomal sorting complex required for transport (ESCRT) have been identified in ALS and FTLD which prevent the formation of the amphisomes, the vesicles that arise from the fusion of the autophagosome with the endosome and ultimately fuse with the lysosome.

Importantly, the many of the details of the autophagosomelysosome fusion process remain unknown. It is likely that additional proteins are involved in this step of autophagy and their mutation/deletion would likely affect autophagosome turnover.

\section{Autophagosomal Clearance}

Once autophagosomes have fused with lysosomes the process of degradation of the cytosolic cargo occurs. Various neurodegenerative diseases are characterized by reduced autophagic flux (increased numbers of autophagosomes). Inefficient autophagosomal clearance can be associated with reduced lysosomal acidification, which reduces the activity of lysosomal hydrolases and promotes the accumulation of undigested byproducts. This is typical of the so-called "lysosomal storage disorders" (LSD), which comprise more than 50 diseases and represent the most common forms of neurodegenerative disease in childhood. While the proteins and mechanisms involved in these diseases differ, impaired autophagy is unfailingly present (Menzies et al., 2015). For example, some forms of PD are characterized by lysosomal dysfunction, which is caused by loss of function mutations in the gene coding for the glucocerebrosidase. This promotes the accumulation of glucocerebroside together with $\alpha$-synuclein in the brain, thus promoting neurodegeneration (Mazzulli 
et al., 2011; Murphy et al., 2014). In AD, however, decreased autophagosomal clearance is associated with mutations in the protein presenilin 1, which increases lysosomal $\mathrm{pH}$, reduces lysosomal activity, thereby promoting amyloid deposition and the onset of the disease (Cataldo et al., 2004).

\section{TDP-43 AND C9orf72: ROLES IN NEURODEGENERATIVE DISEASES}

\section{The TDP-43 Protein}

TAR DNA-binding protein (TARDBP), hereafter called TDP-43, was initially described as a transcriptional repressor factor of HIV (Ou et al., 1995). Since then, numerous studies have established that TDP-43 is a RNA binding protein that participates in multiple pathways of the "RNA metabolism," which includes RNA splicing, RNA transport, mRNA stability, microRNA processing and stress granules assembly (Aulas et al., 2012; Buratti and Baralle, 2012; Ratti and Buratti, 2016) (Figure 3A). From a molecular point of view, the N-terminal of TDP-43 contains a nuclear localization signal (NLS), a nuclear exclusion signal (NES) and two RNA binding domains (RMM1, RMM2). The presence of NLS and NES allows the shuttling of TDP-43 between the nucleus and the cytoplasm (Ayala et al., 2008; Prpar Mihevc et al., 2016). The RRM1 is the region through which TDP-43 binds different RNAs targets (Buratti and Baralle, 2001; Ayala et al., 2011; Avendaño-Vázquez et al., 2012). Additional studies have suggested that RRM2 is required to bind the DNA and to maintain the protein structure (Kuo et al., 2009). Finally, the C-terminal region of TDP-43 is composed by a glycine rich domain (GRD), which has been involved in most of proteinprotein interactions (Buratti et al., 2005; D'ambrogio et al., 2009; Buratti and Baralle, 2012). The GRD domain includes a glutamine/asparagine $(\mathrm{Q} / \mathrm{N})$ prion-like domain responsible for both, protein-protein interactions (Budini et al., 2012a) and TDP43 aggregation (Fuentealba et al., 2010; Budini et al., 2012a,b). In addition to the $\mathrm{Q} / \mathrm{N}$ region, also the $\mathrm{N}$-terminal region of TDP-43 has been implicated in the aggregation process. Indeed, this domain is required to sequester the native TDP-43 into the aggregates, causing a loss-of-function (Figure 3B) (Budini et al., 2015; De Conti et al., 2015; Romano et al., 2015; Langellotti et al., 2016).

TDP-43 is the major component of inclusions or aggregates present in neuronal cells from patients affected by ALS and FTLD (Arai et al., 2006; Neumann et al., 2006). Studies have shown that more than forty disease-associated mutations have been discovered in TDP-43, principally in the C-terminal region of the protein (Buratti and Baralle, 2009; Mackenzie et al., 2010; Lattante et al., 2013). Even though experimental data suggest that some TDP-43 mutations favor its aggregation (Budini et al., $2012 \mathrm{~b}$ ), it is worth to note that around the $95 \%$ of people showing TDP-43 inclusions the protein is not mutated (Lattante et al., 2015). Hence, TDP-43 aggregation process does not necessarily require protein mutations.

As previously mentioned, TDP-43 shuttles between the nucleus and the cytoplasm (Ayala et al., 2008), however, in pathological conditions, it has been observed that TDP-43 mainly remains in the cytoplasm where it can aggregate. Indeed, affected cells can show cytoplasmic TDP-43 aggregates and complete depletion of TDP-43 in the nuclear compartment (Arai et al., 2006; Neumann et al., 2006). TDP-43 aggregates are mainly composed by TDP-43 full-length and by C-terminal protein fragments $(25$ and $35 \mathrm{kDa})$ that can be distinguished by ubiquitination and specific phosphorylation (Arai et al., 2006; Neumann et al., 2006; Hasegawa et al., 2008; Inukai et al., 2008).

On the other hand, mitochondrial dysfunction, observed in samples from patients with ALS, may be regulated by TDP-43. Indeed, overexpression of TDP-43 and its C-terminal fragments induced mitochondrial damage in NSC-34 motor neuron cell line (Hong et al., 2012). In addition, full-length TDP-43 and truncated TDP-43 localize in the mitochondria, suggesting that TDP-43 and its C-terminal fragments cause mitochondrial dysfunction and enhance mitophagy (Hong et al., 2012). These observations were also confirmed by Onesto et al. (2016), who reported that the overexpression of a pathological A382T TDP43 mutant in fibroblasts, induced changes in mitochondria morphology which caused the fragmentation of the mitochondria network (Onesto et al., 2016). Thus, since mitochondria are key in neuronal metabolism, TDP-43 mutations can induce cell death by impairing not only TDP-43-dependent RNA metabolism, but also mitochondria function in ALS. In line with this, it was demonstrated that lithium prevents most ALS pathological changes in vitro an in vivo models by mechanisms that involve mitochondrial protection, induction of autophagy, mitophagy and mitochondriogenesis (Sarkar et al., 2005; Tasdemir et al., 2007; Pasquali et al., 2009; Natale et al., 2015).

Currently, the role of TDP-43 aggregates in neurodegenerative diseases is unknown and two hypotheses have been proposed (Lee et al., 2012): (1) the "gain-of-function" hypothesis, which suggests that cytoplasmic TDP-43 aggregates, or the presence of missense mutations in TDP-43, provokes cell death by cytotoxic effects (Johnson et al., 2008, 2009; Zhang et al., 2009); (2) the "loss-of-function" hypothesis, which suggests that TDP-43 aggregates act as a "sink" by additionally sequestering functional TDP-43 (nuclear and cytoplasmic). This causes a generalized impairment in TDP-43 activity that affects the whole cell physiology (Figure 3B). Despite studies strongly support the idea that the aggregation induces TDP-43 loss-of-function (Budini et al., 2015; De Conti et al., 2015; Romano et al., 2015), it is still not clear which hypothesis is correct and, it is highly possible that a combination of both mechanisms, gain- and loss-of-function, contributes to the onset and disease progression.

\section{The C9orf72 Genetic Factor}

In addition to TDP-43, other genetic markers have been associated with the onset of ALS and FTLD and, among those, the most studied is the genetic factor C9orf72 (Lattante et al., 2015). This factor, was described in 2011 by two independent groups and consists of a polymorphism composed by different repeats of the hexanucleotide GGGGCC $\left[\left(\mathrm{G}_{4} \mathrm{C}_{2}\right) \mathrm{n}\right]$. These repeats locate in a non-coding region of $\mathrm{C} 9$ orf72, specifically 

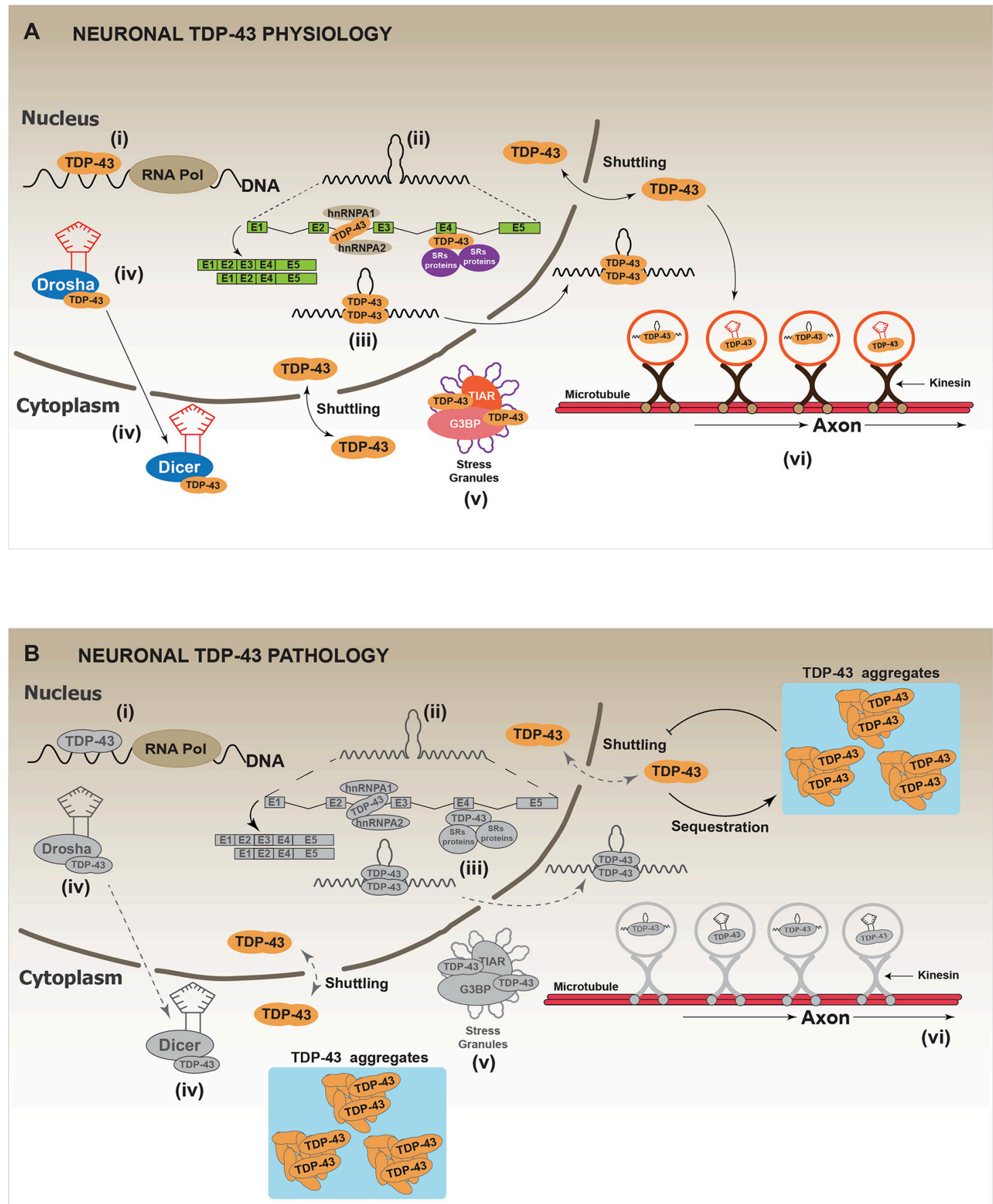

FIGURE 3 | Physiology and pathology of TDP-43. (A) In physiological conditions, thanks to its capacity to shuttle between the nucleus and the cytoplasm, TDP-43 regulates several processes that impact on the cellular fate of different RNAs. These processes are: (i) RNA transcription: it includes the regulation of RNA transcription through the direct binding of TDP-43 to promoter regions. (ii) mRNA alternative splicing: the most studied and known function of TDP-43 is the regulation of alternative mRNA splicing. TDP-43 binds to exon or intron regions and recruits other RNA binding proteins (RBPs) such as hnRNPs or SRs proteins (heterogeneous nuclear ribonucleoproteins and serine-arginine proteins, respectively). (iii) RNA stabilization: RNA stabilization has been proposed as a function of TDP-43. TDP-43, binding to different mRNAs or miRNAs inhibits their premature degradation and possibly favors their translocation from the nucleus to the cytoplasm. Different studies report a decrease in the half-life of several mRNAs and miRNAs after TDP-43 down-regulation. (iv) miRNAs synthesis and processing: TDP-43 has been implicated in the synthesis and processing of different miRNAs by interacting with Drosha and Dicer complexes. It has been reported that TDP-43 down-regulation affects the cellular levels of several miRNAs. (v) Stress granules localization/regulation: In different conditions of cellular stress, it has been shown that TDP-43 is located in the stress granules (SGs) where it interacts with classical SGs proteins such as TIAR and G3BP. Although the real function of TDP-43 in SGs is still unknown, it has been 


\section{FIGURE 3 | Continued}

reported that TDP-43 down-regulation affects SGs formation by decreasing G3BP mRNA levels. (vi) RNA transport: Data indicate TDP-43 is required for the transport of different RNAs along the neuronal axon, by a mechanism that might involve microtubules and kinesin complexes. (B) In a pathological situation, TDP-43 forms cellular aggregates, mainly in the cell cytoplasm, through a mechanism that is currently unknown. It has been proposed that TDP-43 aggregates can be toxic for the cell. However, a generally accepted hypothesis suggests that TDP-43 aggregates could act as "sink" that continuously sequesters functional TDP-43 protein, thus, increasing the aggregation process by a positive feed-back loop. This would lead to a generalized TDP-43 loss-of-function, which promotes cellular stress and eventually leads to cell death. Gray color: represents attenuated processes due to TDP-43 loss-of-function.

within the intron that separates exons $1 \mathrm{a}$ and $1 \mathrm{~b}$ (DeJesusHernandez et al., 2011; Renton et al., 2011). It has been shown that healthy people carry 20 or less $\mathrm{G}_{4} \mathrm{C}_{2}$ repeats $(n \leq 20)$, whereas affected individuals incorporate between 30 and 250 hexanucleotide repeats $(n \geq 30)$ (Renton et al., 2011). The C9orf72 gene can generate three different variants by alternative splicing of its pre-mRNA (DeJesus-Hernandez et al., 2011). By analyzing brain samples from patients, studies indicated that the pathological mechanism of $\left(\mathrm{G}_{4} \mathrm{C}_{2}\right)$ n repeats may involve both, a reduction in the expression of some C9orf72 variants and/or the aberrant formation of RNA foci (DeJesus-Hernandez et al., 2011). Additionally, studies performed during the last 5 years indicated that RNA foci are formed by stable multimeric G-quadruplexes, which can abnormally sequester RNA-binding proteins (RBPs) impairing their physiological functions. In addition, RNA foci can also suffer repeat-associated non-ATG (RAN) translation. This translation occurs in sense and antisense of the $\left(\mathrm{G}_{4} \mathrm{C}_{2}\right)$ n repeats, in all reading frames, and in the absence of an initiating ATG codon. Thus, this mechanism can generate polypeptides formed by different numbers of dipeptide units originated from one $\left(\mathrm{G}_{4} \mathrm{C}_{2}\right) \mathrm{n}$ repeat. These polypetides, known as DRPs (Dipeptide Repeat Proteins) or C9RANT proteins, have shown to be toxic for the cell, since they impair RNA biogenesis ( $\mathrm{Zu}$ et al., 2013; Kwon et al., 2014; Gendron et al., 2015). Studies have evaluated the effects of proline/arginine repeated 20 times (PR20) RAN translation products in primary neuronal cultures of rat spinal cord. It has been shown that PR20 induces cell death in motor neurons without causing mitochondrial alterations, ER stress or mTOR inhibition (Gupta et al., 2017). Additionally, PR20 accumulation inhibits protein degradation pathways, such as autophagy and the ubiquitin proteasome system (Gupta et al., 2017). The pathological effects of $\left(\mathrm{G}_{4} \mathrm{C}_{2}\right) \mathrm{n}$ repeats have been recently reviewed by Todd and Petrucelli (2016).

The physiological role of C9orf72 has been poorly studied. Two different groups found that C9orf72 shares structural similarities with proteins containing DENN modules (Zhang et al., 2012; Levine et al., 2013). Proteins containing DENN modules work as GDP-GTP exchanging factors for RAB GTPases, which are involved in endosomal traffic and in the formation of autophagic vacuoles (Zerial and McBride, 2001; Amaya et al., 2015). Thus, it was proposed that C9orf72 has a role in both endosomal traffic and autophagy. Additional studies showed that C9orf72 interacts with different RAB proteins (Farg et al., 2014) involved in the autophagic process, confirming the hypothesis that C9orf72 plays an important role in the autophagic pathway (Amaya et al., 2015).

\section{ROLE OF AUTOPHAGY IN TDP-43 AND C9orf72 RELATED DISEASES}

\section{Impaired Autophagy in ALS and FTLD}

Studies have demonstrated that mutations in autophagy-related genes are associated with familial ALS and FTLD (Skibinski et al., 2005; Parkinson et al., 2006; Lee et al., 2009; Maruyama et al., 2010; Le Ber et al., 2013; Shimizu et al., 2013; Teyssou et al., 2013; Wong and Holzbaur, 2014; Cirulli et al., 2015; Krasniak and Ahmad, 2016). In fact, mutations in the ubiquitin-binding site on P62/SQSTM1 have been associated with ALS and FTLD caused by the inhibition of protein aggregates clearance mediated by autophagy (Le Ber et al., 2013; Shimizu et al., 2013; Teyssou et al., 2013). Other genes, whose mutations are associated with impaired autophagy regulation, are Optineurin (optn) and CHMP2B. OPTN, which is involved in the mitophagy machinery, is proposed to impact on ALS as its mutations promote severe mitochondria degradation (Maruyama et al., 2010; Wong and Holzbaur, 2014). CHMP2B, which is part of the ESCRTIII protein complex, is involved both in the endosomal-sorting pathway (Skibinski et al., 2005; Parkinson et al., 2006) and in the formation of autophagolysosomes (Filimonenko et al., 2007). In agreement with this, it has been observed that mutations in CHMP2B promote autophagic vesicles accumulation in brain tissues of FTLD patients (Lee et al., 2009; Krasniak and Ahmad, 2016).

Furthermore, new genetic factors that associate the autophagic machinery and neurodegenerative diseases have been identified. A recent exome sequencing assay performed on more than 2,000 ALS patients identified the TANK-Binding Kinase 1 (Tbk1) as a new gene associated with ALS (Cirulli et al., 2015). TBK1 protein belongs to the IKK-kinase family, which is involved in immune signaling pathways in neurons and other cell types (Oakes et al., 2017). In this regard, studies have suggested that TBK1 may be implicated in protein aggregations-induced neuroinflammation (Oakes et al., 2017). Other studies have shown that TBK1 phosphorylates OPTN and p62/SQSTM1 to promote autophagy, and, when non-functional, TBK1 can impair autophagy and mitophagy, affecting neuronal homeostasis (Matsumoto et al., 2015; Richter et al., 2016).

Altogether, these studies suggest that mutations in autophagyrelated genes and, as consequence, impaired autophagy in the brain tissue might contribute to the onset or progression of ALS and FTLD.

\section{Autophagy AND TDP-43 \\ Regulation of TDP-43 by Autophagy}

Studies have shown that autophagy vacuole proteins such as LC3 and p62/SQSTM1 co-localize with TPD-43 aggregates, 
supporting the hypothesis that physiological rates of autophagy are required to avoid the accumulation of TDP43 aggregates (Mizuno et al., 2006; Hiji et al., 2008). Indeed, it was demonstrated, using different approaches, that TDP-43 inclusions on spinal cord samples from ALS and FTLD patients are also positive for p62/SQSTM1 (Mizuno et al., 2006; Hiji et al., 2008). In addition, other studies have suggested that the co-localization between TDP-43, p62/SQSTM1 and ubiquitin is common in sporadic and familial ALS cases as well as in other TDP-43 proteinopathies (Maekawa et al., 2009; King et al., 2011).

In addition to the autophagic vacuole markers p62/SQSTM1 and LC3, also autophagy regulator proteins have been identified co-localizing with TDP-43 inclusions. This is the case of Vasolincontaining protein (VCP) and OPTN, which co-localize with TDP-43, p62/SQSTM1 and ubiquitin in spinal motor neurons of sporadic ALS patients (Ayaki et al., 2014). Thus, the identification of vacuole autophagy markers or autophagy regulator proteins in TDP-43 inclusions suggests that autophagy can be up-regulated to prevent the pathological accumulation of TDP-43 aggregates.

Given that protein inclusions co-localize with autophagic vacuole markers in samples from patients affected by TDP-43dependent proteinopathies, different groups have evaluated whether autophagy regulates the formation of TDP-43 aggregates. Caccamo et al. (2009) showed that inhibition of autophagy by treatment with 3-Methyladenine (3-MA), promotes the accumulation of full-length TDP-43 and its 35 and $25 \mathrm{kDa}$ degradation products in N2a and SH-SY5Y cells. Similar results were observed in HEK-293 cells overexpressing GFP-TDP-43 WT or the GFP-TDP-25 kDa fragment, since the treatment with 3-MA increased the levels of the exogenous forms of TDP-43. Consistently, when autophagy was induced in N2a and SH-SY5Y cells by treatment with rapamycin, the degradation of the different forms of TDP-43 was enhanced (Caccamo et al., 2009).

Elevated expression of LC3 has been identified in skin biopsies of ALS patients carrying the TDP-43 A315T mutation, suggesting that this pathogenic TDP-43 mutation might induce autophagy (Wang et al., 2015). In line with this study, Barmada et al. screened over a million of compounds and found that pluphenazine-, methontrimeprazine- and compound $10-\left(4^{\prime}-(\mathrm{N}-\right.$ diethylamino)butyl)-2- chlorophenoxazine are able to induce autophagy, enhance the turnover of TDP-43 WT and TDP-43 A315T, and increase the survival of neurons in ALS models (Barmada et al., 2014).

The control of TDP-43 protein levels by autophagy can also be co-regulated by heat shock proteins (HSP). Indeed, it has been observed that HSP-90, cell division cycle 37 (CDC37) and TDP43 form a protein complex that prevents TDP-43 degradation in HeLa cells. The disruption of this complex, by downregulation of HSP-90 or CDC37 promoted degradation of TDP-43 via induction of autophagy (Jinwal et al., 2012). The same results were found in the neuronal-like cell lines NSC-43 and SH-SY5Y (Crippa et al., 2010a,b, 2016), in which overexpression of the small heat shock protein B8 (HspB8) induced the degradation of TDP-43 WT and its truncated forms, TDP-43 $\Delta \mathrm{C}$ and TDP$25 \mathrm{kDa}$ by increasing autophagy (Crippa et al., 2010a). Notably, colchicine- and doxorubicin-induced up-regulation of HspB8, enhanced the expression of $t f e b, p 62 / s q s t m 1$ and $l c 3$, suggesting that HspB8 might have a pro-autophagic role, which can be associated to TDP-43 catabolism (Crippa et al., 2016).

As previously mentioned, another adaptor protein involved in TDP-43 degradation through autophagy is OPTN. As indicated, mutations in the optn gene have been found in ALS patients (Shen et al., 2015). Consistently, in N2a cells, mutations affecting the ubiquitin binding site of OPTN enhance its accumulation in inclusion bodies (IBs), where TDP-43 has also been identified. These mutations also modify the degradation rate of TDP-43 through autophagy, probably because mutant proteins are unable to interact with myosin VI (MYO6) and with the target of Myb1 membrane trafficking protein (TOM1), thus avoiding the process of autophagosomes maturation (Shen et al., 2015).

Additionally, studies have demonstrated that autophagy and the ubiquitin proteasome system (UPS) are strictly coordinated to degrade WT and aggregated forms of TDP-43 (Urushitani et al., 2010; Wang et al., 2010; Huang et al., 2014). In accordance with this, studies in HEK-293 and SH-SY5Y cells showed that even though WT TDP-43 is mainly degraded by the UPS system, aggregated forms of TDP-43 are preferentially degraded by autophagy (Scotter et al., 2014). Interestingly, the inhibition of both UPS and autophagy induces the aggregation of $\triangle$ NLS-TDP43 , a mutant form of TDP-43 unable to localize at the cell nucleus. These data suggest that a combination between an excessive accumulation of cytoplasmic TDP-43 and the impairment in cellular degradative pathways leads to TDP-43 aggregation. Thus, given that more than one protein turnover pathway is involved in the catabolism of TDP-43, it seems that the recycling of TDP-43 must be tightly controlled (Ayala et al., 2011; Avendaño-Vázquez et al., 2012).

In vivo, the role of autophagy in the regulation of TDP43 levels has been poorly analyzed. Studies showed that rapamycin induced-autophagy improved learning and memory in transgenic mice overexpressing WT TDP-43. Treatment with rapamycin, which inhibits mTOR activity, increased LC3 I to LC3 II conversion and the turnover of different forms of TDP-43 (full length, 25 and $35 \mathrm{kDa}$ ) (Wang et al., 2012). These studies further support data showing that autophagy is involved in the turnover of full length and aggregated forms of TDP-43.

Altogether, these studies indicate that autophagy is implicated in the control of TDP-43 protein levels, affecting both WT and the aggregated forms of TDP-43 in vitro and in vivo. Thus, autophagy is crucial in the onset and progression of TDP-43related neurodegenerative diseases. The discovery of new drugs able to modulate autophagy needs to be considered as a strategy for the treatment of these diseases associated with dysregulated proteostasis of TDP-43.

\section{Regulation of Autophagy by TDP-43}

As previously mentioned, TDP-43 is implicated in the regulation of autophagy. Indeed, mRNA hybridization assays in N2a cells showed that TDP-43 binds to atg7 mRNA through its RRM1 domain and that down-regulation of TDP-43 decreased atg7 mRNA levels (Bose et al., 2011). Consistently, cells lacking TDP43 showed reduced ATG7 protein levels compared to wild-type cells, as well as impairment in basal and stimulated autophagy, 
as indicated by a reduction in LC3-II and accumulation of p62/SQSTM1, autophagosomes and ubiquitinated inclusions (Bose et al., 2011).

Recently, Xia et al. showed that TDP-43 down-regulation in HeLa, HEK293, HT22, PC12, and SH-SY5Y cell lines, promotes the nuclear translocation of Transcription Factor EB (TFEB), a master transcription factor that regulates the expression of genes controlling lysosomes biogenesis and autophagy induction (Napolitano and Ballabio, 2016; Xia et al., 2016). In this study authors also reported that TDP-43 controls the level of RAPTOR, which is required to maintain the activity of mTOR, by stabilizing its mRNA (Xia et al., 2016). Thus, silencing of TDP43 inactivated mTOR by decreasing RAPTOR levels (Xia et al., 2016). Given that phosphorylation of TFEB by mTOR is required to maintain the cytoplasmic location of TFEB, down-regulation of TDP-43 decreased mTOR-dependent TFEB phosphorylation and induced the translocation of TFEB into the nucleus. The augmented nuclear localization of TFEB increased the gene expression of lamp1, lamp2, atg5, beclin-1, cathepsinL among others autophagy related genes (Xia et al., 2016). In accordance with this, LC3-II, lysosomes and autophagosomes were also accumulated (Xia et al., 2016). Despite these data, this study also found that down-regulation of TDP-43 induced accumulation of p62/SQSTM1, suggesting that, TDP-43 is also required for an optimal autophagosome-lysosome fusion (Xia et al., 2016). A possible explanation for these observations is that TDP-43 is also necessary to stabilize the mRNA of Dynactin 1, which participates in autophagosome-lysosome fusion (Xia et al., 2016). Thus, TDP-43 down-regulation decreases Dynactin 1 protein levels and, as consequence, it impairs autophagosome-lysosome fusion (Xia et al., 2016). In summary, these data indicate that, down-regulation of TDP-43 promotes autophagy through the transcriptional role of nuclear TFEB. However, because TDP43 is also required for autophagosome-lysosome fusion by regulating Dynactin 1, the lack of TDP-43 finally causes an impairment in the progression of the autophagic flux (Xia et al., 2016). Figure 4 summarizes the role of TDP-43 in the regulation of the autophagic process.

As mentioned before, studies strongly suggest that TDP-43 aggregates conduce to a TDP-43 loss-of-function (Budini et al., 2015; De Conti et al., 2015). In this condition, the lack of TDP43 activity may increase cellular stress by inhibiting autophagy. Considering this scenario, the lack of autophagy will further enhance the accumulation of TDP-43 aggregates, leading to an increase in cellular stress, cell death and neurodegeneration (Figure 5). These results are supported by data obtained using a transgenic mouse model which overexpresses the $25 \mathrm{kDa}$ TDP43 fragment in the brain and in the spinal cord. This transgenic mouse showed reduced autophagy and cognitive alterations as suggested by a decrease in different autophagic markers, such as ATG3, ATG7, LC3-II, p62/SQSTM1 and Beclin 1 (Caccamo et al., 2015).

\section{Autophagy and C9orf72}

\section{Regulation of Autophagy by C9orf72}

The physiological functions of C9orf72 protein have been poorly studied. However, studies published during the last years suggest that C9orf72 plays an important role in the regulation of autophagy, thus impacting the neurodegeneration process. Indeed, ALS and FTLD patients carrying C9orf72 repeats show altered autophagy. Immunohistochemical analysis of 36 patients affected by ALS and FTDL showed a significant accumulation of p62/SQSTM1 in neuronal cells (Al-Sarraj et al., 2011). In addition, tissues from cerebral cortex, basal ganglia, hippocampus and cerebellum from ALS patients showed a strong reactivity for p62/SQSTM1 in association with C9orf72 foci. However, the evaluated samples were negative for TDP43 inclusions (Cooper-Knock et al., 2012; Troakes et al., 2012). The same results were observed in FTLD patients, in which the hippocampus, cerebellum, frontal and temporal cortex tissues showed accumulated p62/SQSTM1 and C9orf72 foci (Fratta et al., 2013). Furthermore, co-localization between C9orf72 and p62/SQSTM1 was also observed in pluripotent stem cellderived neurons from patients having a pathological number of $\mathrm{G}_{4} \mathrm{C}_{2}$ repeats. Interestingly, reduced cell viability was also observed in these cells (Almeida et al., 2013). Altogether, these studies indicated that the pathological number of $\mathrm{G}_{4} \mathrm{C}_{2}$ repeats in C9orf72 is associated with impaired autophagic flux.

In addition to the clinical observations, bioinformatic studies showed that C9orf72 protein contains a DENN module (Zhang et al., 2012), which works as a GDP-GTP exchange factor for RAB-GTPases involved in membrane trafficking and autophagy (Levine et al., 2013). Consistently, experiments performed on ALS patient samples, neuronal cell lines (N2a, SH-SY5Y) and primary neuronal cell cultures indicated that the C9orf72 protein co-localizes and interacts with RAB1, RAB5, RAB7, and RAB11, which are involved in autophagy (Farg et al., 2014). Furthermore, C9orf72 also locates in autophagosomes and lysosomes, as demonstrated in cells transfected with LC3-DsRed or dyed with Lysotracker. These results indicate that C9orf72 directly affects the autophagic machinery (Farg et al., 2014). Indeed, studies performed in HEK-293, HeLa, SH-SY5Y, primary cortical neurons and induced neuronal progenitor cells (iNPC) have shown that small and large variants of C9orf72 interact with the ULK complex, inducing activation and translocation of ULK to the phagophore assembly site, thus up-regulating autophagy (Webster et al., 2016).

As mentioned before, the interaction of $\mathrm{C} 90 \mathrm{orf} 72$ with specific RABs or with the ULK complex positively regulates autophagy (Farg et al., 2014). However, recent studies have shown that C9orf72 has to bind additional key proteins to promote autophagy. In this context, three independent works demonstrated, by affinity purification and mass spectrometry analyses in different cell lines (N2a and HEK-293) that the large variant of C9orf72 interacts with WDR41 and SMCR8 (Sellier et al., 2016; Sullivan et al., 2016; Ying et al., 2016). WDR41 and SMCR8 are two DENN proteins identified as part of the human autophagic interactome (Behrends et al., 2010). These studies showed that the C9orf72/SMCR8/WDR41 complex is required for the recruitment of the autophagic initiation complex FIP200/ULK-1/ATG13/ATG10 and RAB8a/RAB39b for the subsequent formation of autophagosomes (Sellier et al., 2016; Sullivan et al., 2016; Yang et al., 2016). In addition, it was also observed that the C9orf72/SMCR8 heterodimer 


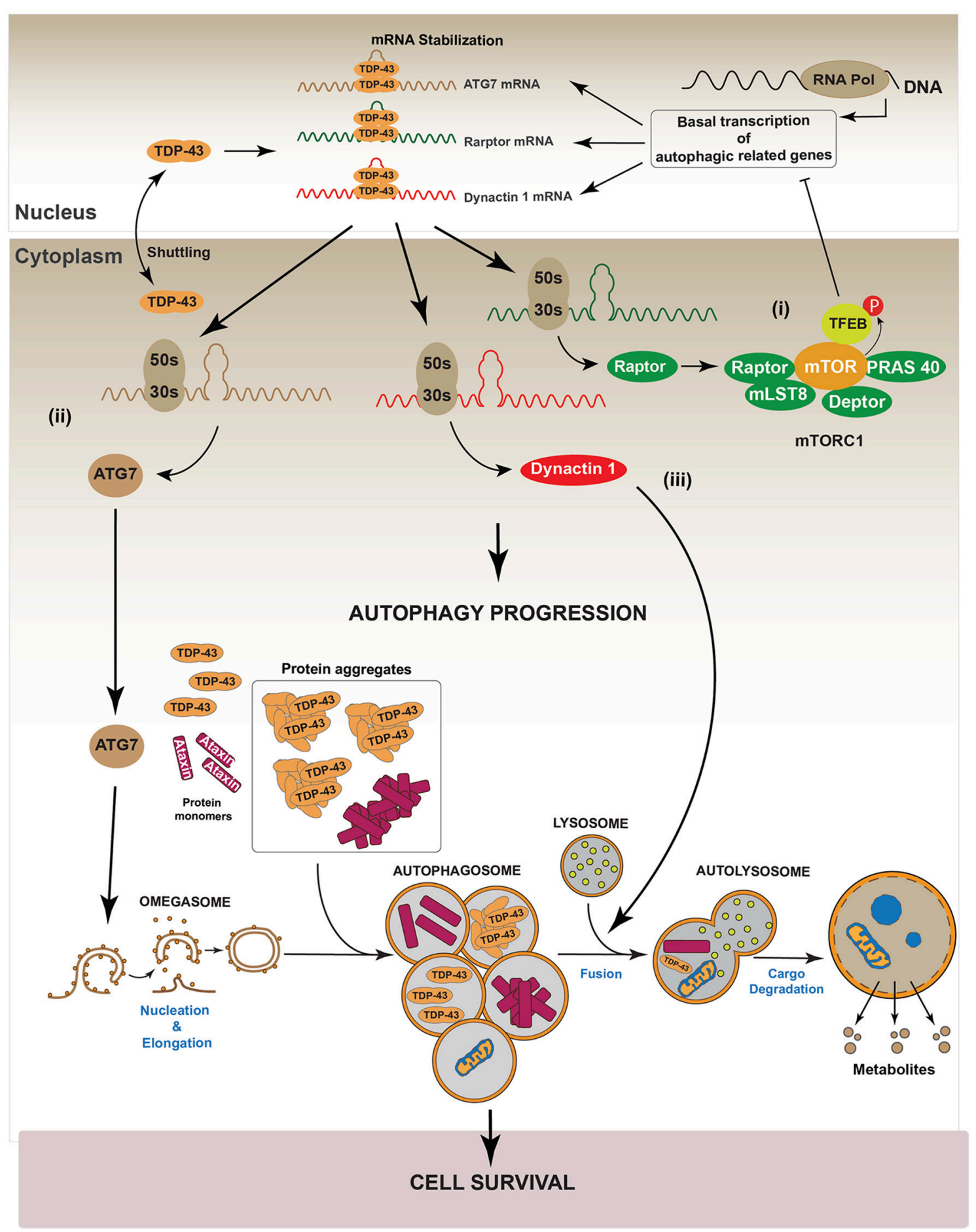

FIGURE 4 | Regulation of autophagy by TDP-43. It has been reported that TDP-43 regulates basal autophagy by stabilizing three different mRNAs: atg7, raptor, and dynactin 1. The cellular levels of these autophagic mRNAs decrease after TDP-43 down-regulation. Thus, data indicate TDP-43 can participate in autophagy regulation at two different steps: autophagic initiation and autophagic flux progression. (I) Autophagy initiation: TDP-43 stabilizes the mRNA that codes for the protein Raptor, thus ensuring sufficient levels of this protein and keeping mTORC1 active (i). In turn, mTORC1 allows the phosphorylation of TFEB decreasing its nuclear translocation. Thus, by stabilizing mRNA raptor levels, TDP-43 ensures the maintenance of basal autophagy. (ii) TDP-43 stabilizes atg7 mRNA and thus its protein levels. In this way, TDP-43 regulates the initiation step and the formation of new autophagosomes (ii). (II) Autophagic flux progression: by stabilizing dynactin $1 \mathrm{mRNA}$ levels and Dynactin 1 protein levels, TDP-43 allows the progression of the autophagic flux (iii). The latter, is supported by data showing that Dynactin 1 is required in the process of autophagosome-lysosome fusion. 


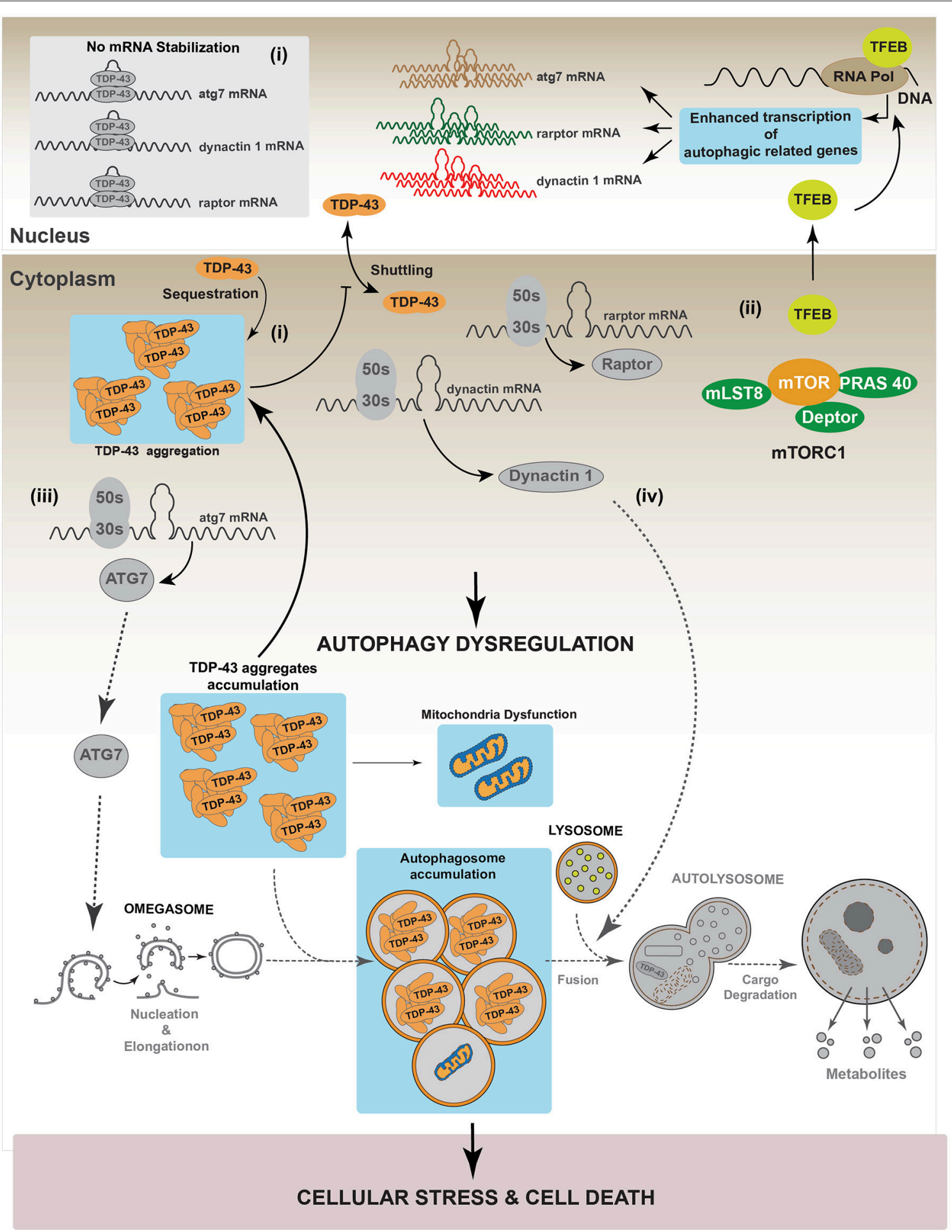

FIGURE 5 | TDP-43 aggregation dysregulates autophagy. In a pathological condition, TDP-43 aggregates might sequester functional TDP-43 causing a complete loss-of-function of this protein. Loss of TDP-43 activity decreases the levels of raptor, atg7 and dynactin 1 mRNAs (i). This situation can affect the autophagic pathway in two different ways. (I) Autophagy induction: loss of Raptor protein destabilizes the mTOR complex decreasing the phosphorylation that inhibits TFEB nuclear translocation. Dephosphorylated TFEB in now able to translocate into the nucleus, where it increases the transcription of genes that stimulate autophagy (ii). Loss of TDP-43 activity negatively impacts on the Atg7 protein level, thus decreasing autophagosomes formation (iii). (II) Autophagy progression: this step can be reduced following TDP-43 aggregation as Dynactin 1 protein levels are lower due to TDP-43 loss-of-function (iv). Overall, although the loss of TDP-43 activity increases the expression of genes related with autophagy progression, autophagy is blocked due to the failure in both, autophagosomes formation and autophagosome-lysosome fusion. This situation can promote cellular stress and cell death. Gray color: represents down-regulated proteins and attenuated processes due to TDP-43 loss-of-function. 


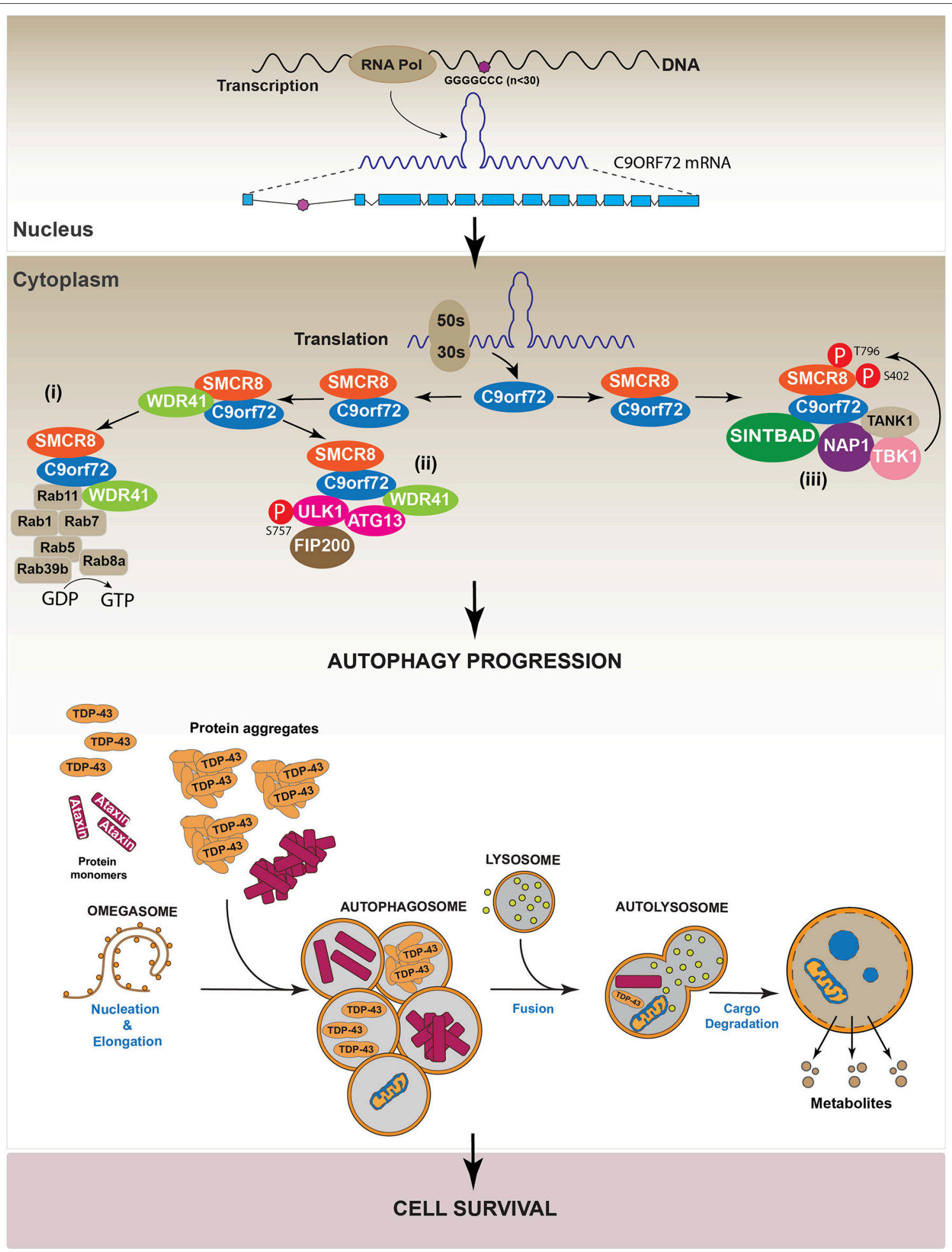

FIGURE 6 | Regulation of autophagy by C9orf72. Studies indicate C9orf72 protein is implicated in autophagy regulation. Specifically, data show C9orf72 is involved in the progression of the autophagic process by three different mechanisms. (i) C9orf72 interacts with SMCR8 forming a heterodimer that recruits WDR4. The complex C9orf72/SMCR8/WDR41 activates several RAB proteins, which participate in autophagy. (ii) The C9orf72/SMCR8/WDR4 complex recruits and activates the ULK complex by its phosphorylation in S575. (iii) The heterodimer C9orf72/SMCR8 interacts with the proteins SINTBAD, NAP, and TANK. These proteins, in turn, recruit TBK1 to the complex allowing the phosphorylation of SMCR8, which is required for autophagy progression. 


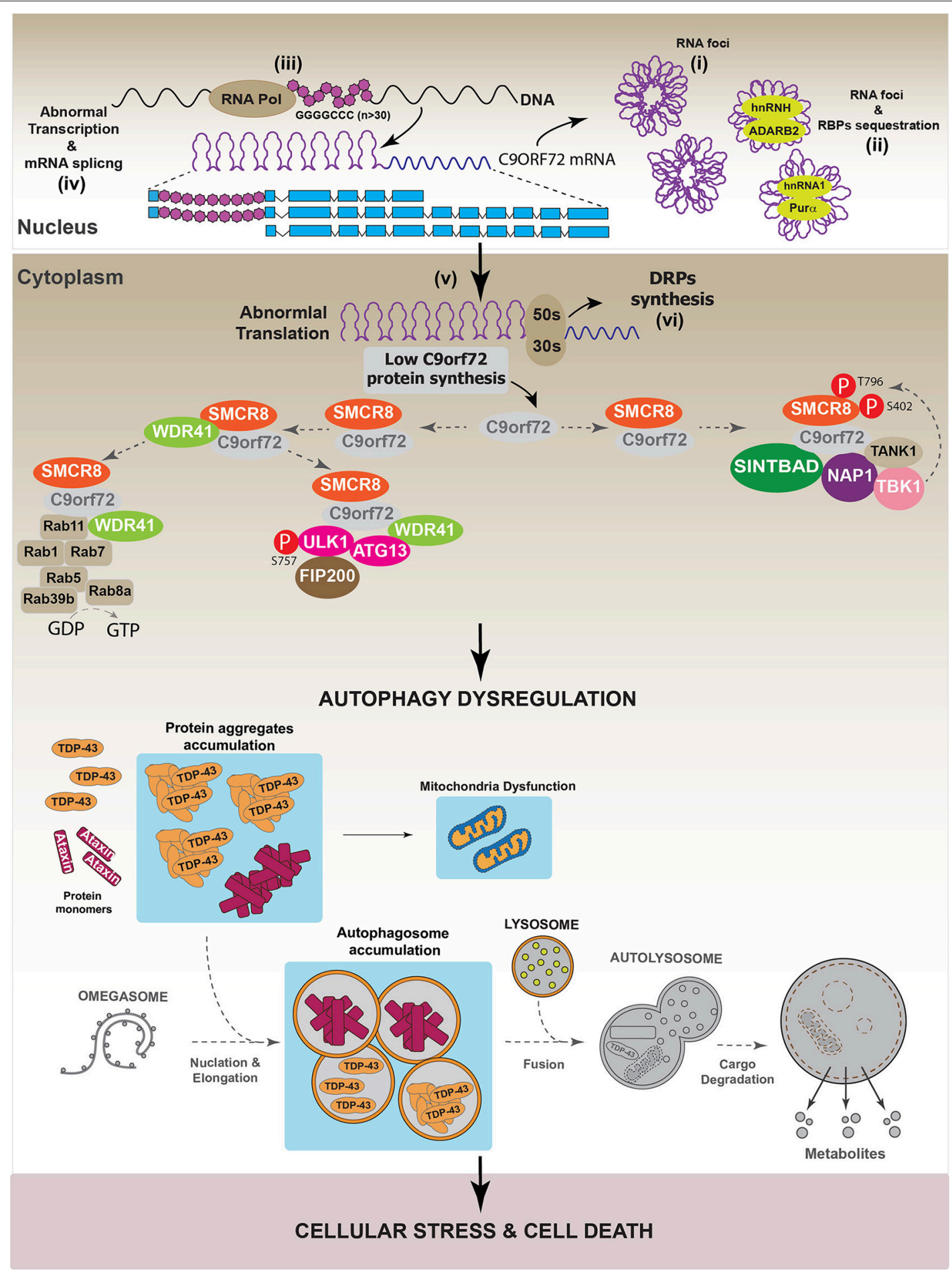

FIGURE 7 | Autophagy dysregulation in C9orf72-dependent neurodegenerative diseases. In a pathological situation, it has been observed that the presence of GGGGCC repeats $(n \geq 30)$ in c9orf72 region can be toxic for the cell. Data indicate that GGGGCC repeats can internally interact to form RNA foci (i) that are toxic by recruiting different RNA binding proteins (RBPs) such as hnRNPH, hnRNPA2, ADARB2, and Pura. (ii) In addition to RNA foci, it has been shown that GGGGCC repeats interfere with the normal transcription (iii), splicing (iv), and translation (v) processes of C9orf72, thus reducing C9orf72 protein levels in the cell. Moreover, several studies showed that GGGGCC repeats can form polypeptides composed by dipeptides repetitions (DRPs). These DRPs are synthetized through a non-classical mechanism of protein translation (vi). To our knowledge, there is no evidence indicating that DRPs might interfere with the autophagic process. Overall, in pathological conditions, GGGGCC repeats decrease C9orf72 protein levels, thus affecting the normal autophagic process. Autophagy dysregulation culminates with the accumulation of autophagosomes, caused by impaired autophagosomal-lysosomal fusion, which might contribute to the formation and accumulation of protein aggregates like Ataxin and eventually TDP-43. This causes cellular stress and might lead to cell death. Gray color: represents down-regulated proteins and attenuated processes due to TDP-43 loss-of-function. 
induced autophagy by phosphorylating ULK at Ser 757. Indeed, down-regulation of C9orf72 decreased the phosphorylation of ULK in MEF cells. Interestingly, this effect was reverted by SMCR8 overexpression, indicating that C9orf72-mediated ULK phosphorylation at Ser 757 is SMCR8 dependent (Yang et al., 2016). Altogether, these studies suggest that C9orf72 regulates autophagy initiation via ULK.

Additionally, data indicate that the C9orf72/SMCR8 complex also interacts with the TRAF Family Member Associated NF $\kappa$ B Activator protein (TANK), TBK1 Binding Protein 1 (SINTBAD) and Nucleosome Assembly Protein 1 (NAP1), forming a protein complex that activates the TANK-Binding Kinase 1 (TBK1). Active TBK1 phosphorylates SMCR8 at Ser 402 and Thr 796, stabilizing the C9orf72/SMCR8 complex and thus inducing autophagy. On the other hand, the interaction between C9orf72/SMCR8/WDR41 and ULK complex induced SMCR8 phosphorylation at Ser 400, 492, 562, and Thr 666/Ser 667. These modifications are less efficient in inducing autophagy compared with those performed by TBK1 (Sellier et al., 2016). Altogether, these studies indicate that C9orf72 is required to form a stable complex with SMCR8 and WDR41, thus allowing the recruitment of different factors such as the ULK complex and RABs to activate the autophagic machinery. The molecular mechanism supporting the new role of C9orf72 in autophagy regulation is summarized in Figure 6.

In agreement with the role of C9orf72 in autophagy, different works have demonstrated that C9orf72 downregulation impaired autophagy progression, as evidenced by the intracellular accumulation of p62/SQSTM1 (Farg et al., 2014; Sellier et al., 2016; Yang et al., 2016). However, it is interesting to note that the dysregulation of the autophagic pathway induced by C9orf72 down-regulation did not induce cell toxicity (Sellier et al., 2016). However, in primary neuronal cultures and in a zebrafish in vivo model, the overexpression of Ataxin-2 Q30 aggregates induced cell toxicity when autophagy was inhibited by C9orf72 down-regulation (Sellier et al., 2016). Altogether, these results suggest that different pathways are implicated in the onset of C9orf72mediated neurodegenerative diseases. One of these promotes the generation of aberrant protein inclusions, such as TDP43 or Ataxin aggregates, while the second one involves the dysregulation of autophagy. To support this hypothesis, it has been observed that C9orf72 down-regulation increased TDP-43 aggregation in E8 mouse cortical neurons, indicating that the pathologies associated with reductions in C9orf72 level promote the onset of diseases characterized by the presence of TDP-43 aggregates (Sellier et al., 2016). Figure 7 summarizes the hypothetical role of C9orf72 in the onset of neurodegenerative diseases.

\section{CONCLUDING REMARKS}

Autophagy dysfunction has been identified in several neurodegenerative diseases such as ALS and FTLD. TDP43 and C9orf72 are also associated with these illnesses as well as other neurodegenerative disorders. TDP-43 aggregates are found in neurons of ALS and FTLD patients, and it has been proposed that they may act as a sink for the newly synthetized TDP-43 protein, thus leading to TDP-43 loss-of-function. Autophagy has been proposed to be crucial for the maintenance of TDP-43 protein levels and to avoid TDP-43 aggregation. Indeed, very recently, data indicate TDP-43 is able to regulate autophagy by stabilizing the mRNA of important components of the autophagic machinery, and, conversely, TDP-43 downregulation/loss-of-function finally inhibits the autophagic flux.

The association of C9orf72 with ALS and FTLD has recently been discovered, and the physiological functions of C9orf72 are still being investigated. Additionally, recent studies showed that C9orf72 is involved in membrane trafficking and autophagy. Specifically, it has been demonstrated that C9orf72 stimulates autophagy initiation by interacting with autophagic proteins involved in membrane trafficking. Consistently, in diseases such as ALS and FTLD, it has been observed a reduction in $\mathrm{C} 9$ orf72 as well as decreased levels of autophagy.

Thus, to date, evidences strongly support that both, TDP43 and C9orf72 regulate autophagy and that loss-of-function of any of these factors blocks the autophagic flux. In the scenario of TDP-43-associated proteinopathies, TDP-43 loss-of-function caused by TDP-43 aggregates, can further increase cellular stress by blocking the autophagic process. In turn, autophagy inhibition provokes an exacerbated accumulation of TDP-43 aggregates, enhances cellular stress and induces cell death, leading to the onset or progression of neurodegenerative diseases.

Consistently, in C9orf72-associated neuropathologies, two events lead to the onset of the disease. The first one is associated with the production of aberrant protein inclusions, such as TDP43 or Ataxin aggregates, while the second one is characterized by the deregulation of autophagy caused by C9orf72 loss-offunction.

Thus, the dysregulation of autophagy is key in the development of TDP-43- and C9orf72-associated neurodegenerative disorders. Even though different are the mechanisms that cause the impairment in autophagy, induction in the autophagic pathway can be considered as a general strategy to avoid these diseases, and research should focus on the identification of novel therapeutic strategies to stimulate autophagy.

\section{AUTHOR CONTRIBUTIONS}

$\mathrm{MB}, \mathrm{EM}$, and $\mathrm{AC}$ wrote the article and made the figures. EB made important contributions, discussions and corrections to the manuscript.

\section{ACKNOWLEDGMENTS}

We thank all members of the Budini and Criollo Lab for helpful insights. We thank our founders and supporters: grants from Fondo Nacional de Desarrollo Científico y Tecnológico, FONDECYT (1161123 to MB, 1160820 to EM, and 1140908 
to AC); by FONDAP-ACCDis (15130011 to AC); by PEW Latin American Fellows Program in the Biomedical Science (to $\mathrm{AC}$ ); by the International Centre for Genetic Engineering and Biotechnology, ICGEB, (CRP/CH113-04RG to MB and CRP/CHL16-06 to EM) and by Ministero Affari Esteri, project SCREENCELLS4ALS (to EB).

\section{REFERENCES}

Almeida, S., Gascon, E., Tran, H., Chou, H. J., Gendron, T. F., Degroot, S., et al. (2013). Modeling key pathological features of frontotemporal dementia with C9ORF72 repeat expansion in iPSC-derived human neurons. Acta Neuropathol. 126, 385-399. doi: 10.1007/s00401-013-1149-y

Al-Sarraj, S., King, A., Troakes, C., Smith, B., Maekawa, S., Bodi, I., et al. (2011). p62 positive, TDP-43 negative, neuronal cytoplasmic and intranuclear inclusions in the cerebellum and hippocampus define the pathology of C9orf72-linked FTLD and MND/ALS. Acta Neuropathol. 122, 691-702. doi: 10.1007/s00401-011-0911-2

Amaya, C., Fader, C. M., and Colombo, M. I. (2015). Autophagy and proteins involved in vesicular trafficking. FEBS Lett. 589, 3343-3353. doi: 10.1016/j.febslet.2015.09.021

Arai, T., Hasegawa, M., Akiyama, H., Ikeda, K., Nonaka, T., Mori, H., et al. (2006). TDP-43 is a component of ubiquitin-positive tau-negative inclusions in frontotemporal lobar degeneration and amyotrophic lateral sclerosis. Biochem. Biophys. Res. Commun. 351, 602-611. doi: 10.1016/j.bbrc.2006.10.093

Atkin, G., and Paulson, H. (2014). Ubiquitin pathways in neurodegenerative disease. Front. Mol. Neurosci. 7:63. doi: 10.3389/fnmol.2014.00063

Aulas, A., Stabile, S., and Vande Velde, C. (2012). Endogenous TDP-43, but not FUS, contributes to stress granule assembly via G3BP. Mol. Neurodegener. 7, 54. doi: 10.1186/1750-1326-7-54

Avendaño-Vázquez, S. E., Dhir, A., Bembich, S., Buratti, E., Proudfoot, N., and Baralle, F. E. (2012). Autoregulation of TDP-43 mRNA levels involves interplay between transcription, splicing, and alternative polyA site selection. Genes Dev. 26, 1679-1684.

Axe, E. L., Walker, S. A., Manifava, M., Chandra, P., Roderick, H. L., Habermann, A., et al. (2008). Autophagosome formation from membrane compartments enriched in phosphatidylinositol 3-phosphate and dynamically connected to the endoplasmic reticulum. J. Cell Biol. 182, 685-701. doi: $10.1083 /$ jcb. 200803137

Ayaki, T., Ito, H., Fukushima, H., Inoue, T., Kondo, T., Ikemoto, A., et al. (2014). Immunoreactivity of valosin-containing protein in sporadic amyotrophic lateral sclerosis and in a case of its novel mutant. Acta Neuropathol. Commun. 2, 172. doi: 10.1186/s40478-014-0172-0

Ayala, Y. M., De Conti, L., Avendano-Vazquez, S. E., Dhir, A., Romano, M., D'Ambrogio, A., et al. (2011). TDP-43 regulates its mRNA levels through a negative feedback loop. EMBO J. 30, 277-288. doi: 10.1038/emboj.2010.310

Ayala, Y. M., Zago, P., D’ambrogio, A., Xu, Y.-F., Petrucelli, L., Baralle, F. E., et al. (2008). Structural determinants of the cellular localization and shuttling of TDP-43. J. Cell Sci. 121, 3778-3785. doi: 10.1242/jcs.038950

Baralle, M., Buratti, E., and Baralle, F. E. (2013). The role of TDP-43 in the pathogenesis of ALS and FTLD. Biochem. Soc. Trans. 41, 1536-1540. doi: 10.1042/BST20130186

Barmada, S. J., Serio, A., Arjun, A., Bilican, B., Daub, A., Ando, D. M., et al. (2014). Autophagy induction enhances TDP43 turnover and survival in neuronal ALS models. Nat. Chem. Biol. 10, 677-685. doi: 10.1038/nchembio.1563

Beghi, E., Logroscino, G., Chio, A., Hardiman, O., Mitchell, D., Swingler, R., et al. (2006). The epidemiology of ALS and the role of population-based registries. Biochim. Biophys. Acta 1762, 1150-1157. doi: 10.1016/j.bbadis.2006.09.008

Behrends, C., Sowa, M. E., Gygi, S. P., and Harper, J. W. (2010). Network organization of the human autophagy system. Nature 466, 68-76. doi: $10.1038 /$ nature09204

Bento, C. F., Renna, M., Ghislat, G., Puri, C., Ashkenazi, A., Vicinanza, M., et al. (2016). Mammalian autophagy: how does it work? Annu. Rev. Biochem. 85, 685-713. doi: 10.1146/annurev-biochem-060815-014556

Birgisdottir, A. B., Lamark, T., and Johansen, T. (2013). The LIR motif - crucial for selective autophagy. J. Cell Sci. 126, 3237-3247. doi: 10.1242/jcs.126128

Bjorkoy, G., Lamark, T., Brech, A., Outzen, H., Perander, M., Overvatn, A., et al. (2005). p62/SQSTM1 forms protein aggregates degraded by autophagy and has a protective effect on huntingtin-induced cell death. J. Cell Biol. 171, 603-614. doi: $10.1083 /$ jcb.200507002

Bodemann, B. O., Orvedahl, A., Cheng, T., Ram, R. R., Ou, Y. H., Formstecher, E., et al. (2011). RalB and the exocyst mediate the cellular starvation response by direct activation of autophagosome assembly. Cell 144, 253-267. doi: 10.1016/j.cell.2010.12.018

Bose, J. K., Huang, C. C., and Shen, C. K. (2011). Regulation of autophagy by neuropathological protein TDP-43. J. Biol. Chem. 286, 44441-44448. doi: 10.1074/jbc.M111.237115

Budini, M., Baralle, F. E., and Buratti, E. (2014). Targeting TDP-43 in neurodegenerative diseases. Expert Opin. Ther. Targets 18, 617-632. doi: $10.1517 / 14728222.2014 .896905$

Budini, M., Buratti, E., Stuani, C., Guarnaccia, C., Romano, V., De Conti, L., et al. (2012a). Cellular model of TAR DNA-binding protein 43 (TDP-43) aggregation based on its C-terminal Gln/Asn-rich region. J. Biol. Chem. 287, 7512-7525. doi: 10.1074/jbc.M111.288720

Budini, M., Romano, V., Avendaño-Vázquez, S. E., Bembich, S., Buratti, E., and Baralle, F. E. (2012b). Role of selected mutations in the Q/N rich region of TDP43 in EGFP-12xQ/N-induced aggregate formation. Brain Res. 1462, 139-150. doi: 10.1016/j.brainres.2012.02.031

Budini, M., Romano, V., Quadri, Z., Buratti, E., and Baralle, F. E. (2015). TDP43 loss of cellular function through aggregation requires additional structural determinants beyond its C-terminal Q/N prion-like domain. Hum. Mol. Genet. 24, 9-20. doi: 10.1093/hmg/ddu415

Buratti, E., and Baralle, F. E. (2001). Characterization and functional implications of the RNA binding properties of nuclear factor TDP-43, a novel splicing regulator of CFTR exon 9. J. Biol. Chem. 276, 36337-36343. doi: 10.1074/jbc.M104236200

Buratti, E., and Baralle, F. E. (2009). The molecular links between TDP-43 dysfunction and neurodegeneration. Adv. Genet. 66, 1-34. doi: 10.1016/s0065-2660(09)66001-6

Buratti, E., and Baralle, F. E. (2012). TDP-43: gumming up neurons through protein-protein and protein-RNA interactions. Trends Biochem. Sci. 37, 237-247. doi: 10.1016/j.tibs.2012.03.003

Buratti, E., Brindisi, A., Giombi, M., Tisminetzky, S., Ayala, Y. M., and Baralle, F. E. (2005). TDP-43 binds heterogeneous nuclear ribonucleoprotein A/B through its C-terminal tail. J. Biol. Chem. 280, 37572-37584. doi: 10.1074/jbc.M505557200

Caccamo, A., Majumder, S., Deng, J. J., Bai, Y., Thornton, F. B., and Oddo, S. (2009). Rapamycin rescues TDP-43 mislocalization and the associated low molecular mass neurofilament instability. J. Biol. Chem. 284, 27416-27424. doi: 10.1074/jbc.M109.031278

Caccamo, A., Shaw, D. M., Guarino, F., Messina, A., Walker, A. W., and Oddo, S. (2015). Reduced protein turnover mediates functional deficits in transgenic mice expressing the $25 \mathrm{kDa}$ C-terminal fragment of TDP-43. Hum. Mol. Genet. 24, 4625-4635. doi: 10.1093/hmg/ddv193

do Carmo Costa, M., and Paulson, H. L., (2013). New hope for therapy in neurodegenerative diseases. Cell. Res. 23, 1159-1160. doi: 10.1038/cr. 2013.96

Cataldo, A. M., Peterhoff, C. M., Schmidt, S. D., Terio, N. B., Duff, K., Beard, M., et al. (2004). Presenilin mutations in familial Alzheimer disease and transgenic mouse models accelerate neuronal lysosomal pathology. J. Neuropathol. Exp. Neurol. 63, 821-830. doi: 10.1093/jnen/63.8.821

Chantranupong, L., and Sabatini, D. M. (2016). Cell biology: The TORC1 pathway to protein destruction. Nature 536, 155-156. doi: 10.1038/nature18919

Ciechanover, A., and Kwon, Y. T. (2015). Degradation of misfolded proteins in neurodegenerative diseases: therapeutic targets and strategies. Exp. Mol. Med. 47, e147. doi: 10.1038/emm.2014.117

Cirulli, E. T., Lasseigne, B. N., Petrovski, S., Sapp, P. C., Dion, P. A., Leblond, C. S., et al. (2015). Exome sequencing in amyotrophic lateral sclerosis identifies risk genes and pathways. Science 347, 1436-1441. doi: 10.1126/science.aaa3650 
Cooper-Knock, J., Hewitt, C., Highley, J. R., Brockington, A., Milano, A., Man, S., et al. (2012). Clinico-pathological features in amyotrophic lateral sclerosis with expansions in C9ORF72. Brain 135, 751-764. doi: 10.1093/brain/awr365

Crippa, V., Carra, S., Rusmini, P., Sau, D., Bolzoni, E., Bendotti, C., et al. (2010a). A role of small heat shock protein B8 (HspB8) in the autophagic removal of misfolded proteins responsible for neurodegenerative diseases. Autophagy 6, 958-960. doi: 10.4161/auto.6.7.13042

Crippa, V., D’Agostino, V. G., Cristofani, R., Rusmini, P., Cicardi, M. E., Messi, E., et al. (2016). Transcriptional induction of the heat shock protein B8 mediates the clearance of misfolded proteins responsible for motor neuron diseases. Sci. Rep. 6:22827. doi: 10.1038/srep22827

Crippa, V., Sau, D., Rusmini, P., Boncoraglio, A., Onesto, E., Bolzoni, E., et al. (2010b). The small heat shock protein B8 (HspB8) promotes autophagic removal of misfolded proteins involved in amyotrophic lateral sclerosis (ALS). Hum. Mol. Genet. 19, 3440-3456. doi: 10.1093/hmg/ddq257

Cruts, M., Gijselinck, I., Van Langenhove, T., van der Zee, J., and Van Broeckhoven, C. (2013). Current insights into the C9orf72 repeat expansion diseases of the FTLD/ALS spectrum. Trends Neurosci. 36, 450-459. doi: 10.1016/j.tins.2013.04.010

D’ambrogio, A., Buratti, E., Stuani, C., Guarnaccia, C., Romano, M., Ayala, Y. M., et al. (2009). Functional mapping of the interaction between TDP-43 and hnRNP A2 in vivo. Nucleic Acids Res. 37, 4116-4126. doi: 10.1093/nar/gkp342

De Conti, L., Akinyi, M. V., Mendoza-Maldonado, R., Romano, M., Baralle, M., and Buratti, E. (2015). TDP-43 affects splicing profiles and isoform production of genes involved in the apoptotic and mitotic cellular pathways. Nucleic Acids Res. 43, 8990-9005. doi: 10.1093/nar/gkv814

DeJesus-Hernandez, M., Mackenzie, I. R., Boeve, B. F., Boxer, A. L., Baker, M., Rutherford, N. J., et al. (2011). Expanded GGGGCC hexanucleotide repeat in noncoding region of C9ORF72 causes chromosome 9p-linked FTD and ALS. Neuron 72, 245-256. doi: 10.1016/j.neuron.2011.09.011

Di Bartolomeo, S., Corazzari, M., Nazio, F., Oliverio, S., Lisi, G., Antonioli, M., et al. (2010). The dynamic interaction of AMBRA1 with the dynein motor complex regulates mammalian autophagy. J. Cell Biol. 191, 155-168. doi: $10.1083 /$ jcb. 201002100

Dodson, M. W., Zhang, T., Jiang, C., Chen, S., and Guo, M. (2012). Roles of the Drosophila LRRK2 homolog in Rab7-dependent lysosomal positioning. Hum. Mol. Genet. 21, 1350-1363. doi: 10.1093/hmg/ddr573

Farg, M. A., Sundaramoorthy, V., Sultana, J. M., Yang, S., Atkinson, R. A., Levina, V., et al. (2014). C9ORF72, implicated in amytrophic lateral sclerosis and frontotemporal dementia, regulates endosomal trafficking. Hum. Mol. Genet. 23, 3579-3595. doi: 10.1093/hmg/ddu068

Fecto, F., Yan, J., Vemula, S. P., Liu, E., Yang, Y., Chen, W., et al. (2011). SQSTM1 mutations in familial and sporadic amyotrophic lateral sclerosis. Arch. Neurol. 68, 1440-1446. doi: 10.1001/archneurol.2011.250

Fernandez, A. F., and Lopez-Otin, C. (2015). The functional and pathologic relevance of autophagy proteases. J. Clin. Invest. 125, 33-41. doi: 10.1172/JCI73940

Ferrucci, M., Fulceri, F., Toti, L., Soldani, P., Siciliano, G., Paparelli, A., et al. (2011). Protein clearing pathways in ALS. Arch. Ital. Biol. 149, 121-149. doi: 10.4449/aib.v149i1.1258

Filimonenko, M., Stuffers, S., Raiborg, C., Yamamoto, A., Malerod, L., Fisher, E. M., et al. (2007). Functional multivesicular bodies are required for autophagic clearance of protein aggregates associated with neurodegenerative disease. J. Cell Biol. 179, 485-500. doi: 10.1083/jcb.200702115

Fraldi, A., Annunziata, F., Lombardi, A., Kaiser, H. J., Medina, D. L., Spampanato, C., et al. (2010). Lysosomal fusion and SNARE function are impaired by cholesterol accumulation in lysosomal storage disorders. EMBO J. 29, 3607-3620. doi: 10.1038/emboj.2010.237

Fratta, P., Poulter, M., Lashley, T., Rohrer, J. D., Polke, J. M., Beck, J., et al. (2013). Homozygosity for the C9orf72 GGGGCC repeat expansion in frontotemporal dementia. Acta Neuropathol. 126, 401-409. doi: 10.1007/s00401-013-1147-0

Fuentealba, R. A., Udan, M., Bell, S., Wegorzewska, I., Shao, J., Diamond, M. I., et al. (2010). Interaction with polyglutamine aggregates reveals a Q/N-rich domain in TDP-43. J. Biol. Chem. 285, 26304-26314. doi: 10.1074/jbc.M110.125039

Gendron, T. F., van Blitterswijk, M., Bieniek, K. F., Daughrity, L. M., Jiang, J., Rush, B. K., et al. (2015). Cerebellar c9RAN proteins associate with clinical and neuropathological characteristics of C9ORF72 repeat expansion carriers. Acta Neuropathol. 130, 559-573. doi: 10.1007/s00401-015-1474-4

Geng, J., Nair, U., Yasumura-Yorimitsu, K., and Klionsky, D. J. (2010). Post-Golgi Sec proteins are required for autophagy in Saccharomyces cerevisiae. Mol. Biol. Cell 21, 2257-2269. doi: 10.1091/mbc.E09-11-0969

Gupta, R., Lan, M., Mojsilovic-Petrovic, J., Choi, W. H., Safren, N., Barmada, S., et al. (2017). The Proline/arginine dipeptide from hexanucleotide repeat expanded C9ORF72 inhibits the proteasome. eNeuro 4, 1-18. doi: 10.1523/ENEURO.0249-16.2017

Gwinn, D. M., Shackelford, D. B., Egan, D. F., Mihaylova, M. M., Mery, A., Vasquez, D. S., et al. (2008). AMPK phosphorylation of raptor mediates a metabolic checkpoint. Mol. Cell 30, 214-226. doi: 10.1016/j.molcel.2008.03.003

Hailey, D. W., Rambold, A. S., Satpute-Krishnan, P., Mitra, K., Sougrat, R., Kim, P. K., et al. (2010). Mitochondria supply membranes for autophagosome biogenesis during starvation. Cell 141, 656-667. doi: 10.1016/j.cell.2010.04.009

Hamasaki, M., Furuta, N., Matsuda, A., Nezu, A., Yamamoto, A., Fujita, N., et al. (2013). Autophagosomes form at ER-mitochondria contact sites. Nature 495, 389-393. doi: 10.1038/nature11910

Hanna, R. A., Quinsay, M. N., Orogo, A. M., Giang, K., Rikka, S., and Gustafsson, A. B. (2012). Microtubule-associated protein 1 light chain 3 (LC3) interacts with Bnip3 protein to selectively remove endoplasmic reticulum and mitochondria via autophagy. J. Biol. Chem. 287, 19094-19104. doi: 10.1074/jbc.M111.322933

Hara, T., Nakamura, K., Matsui, M., Yamamoto, A., Nakahara, Y., SuzukiMigishima, R., et al. (2006). Suppression of basal autophagy in neural cells causes neurodegenerative disease in mice. Nature 441, 885-889. doi: $10.1038 /$ nature 04724

Hara, T., Takamura, A., Kishi, C., Iemura, S., Natsume, T., Guan, J. L., et al. (2008). FIP200, a ULK-interacting protein, is required for autophagosome formation in mammalian cells. J. Cell Biol. 181, 497-510. doi: 10.1083/jcb.2007 12064

Hasegawa, M., Arai, T., Nonaka, T., Kametani, F., Yoshida, M., Hashizume, Y., et al. (2008). Phosphorylated TDP-43 in frontotemporal lobar degeneration and amyotrophic lateral sclerosis. Ann. Neurol. 64, 60-70. doi: 10.1002/ana.21425

Hayashi-Nishino, M., Fujita, N., Noda, T., Yamaguchi, A., Yoshimori, T., and Yamamoto, A. (2009). A subdomain of the endoplasmic reticulum forms a cradle for autophagosome formation. Nat. Cell Biol. 11, 1433-1437. doi: $10.1038 /$ ncb1991

Heras-Sandoval, D., Perez-Rojas, J. M., Hernandez-Damian, J., and PedrazaChaverri, J. (2014). The role of PI3K/AKT/mTOR pathway in the modulation of autophagy and the clearance of protein aggregates in neurodegeneration. Cell. Signal. 26, 2694-2701. doi: 10.1016/j.cellsig.2014.08.019

Hiji, M., Takahashi, T., Fukuba, H., Yamashita, H., Kohriyama, T., and Matsumoto, M. (2008). White matter lesions in the brain with frontotemporal lobar degeneration with motor neuron disease: TDP-43-immunopositive inclusions co-localize with p62, but not ubiquitin. Acta Neuropathol. 116, 183-191. doi: 10.1007/s00401-008-0402-2

Hollenbeck, P. J. (1993). Products of endocytosis and autophagy are retrieved from axons by regulated retrograde organelle transport. J. Cell Biol. 121, 305-315. doi: $10.1083 /$ jcb.121.2.305

Hong, K., Li, Y., Duan, W., Guo, Y., Jiang, H., Li, W., et al. (2012). Full-length TDP-43 and its C-terminal fragments activate mitophagy in NSC34 cell line. Neurosci. Lett. 530, 144-149. doi: 10.1016/j.neulet.2012.10.003

Huang, C. C., Bose, J. K., Majumder, P., Lee, K. H., Huang, J. T., Huang, J. K., et al. (2014). Metabolism and mis-metabolism of the neuropathological signature protein TDP-43. J. Cell Sci. 127, 3024-3038. doi: 10.1242/jcs. 136150

Inoki, K., Li, Y., Zhu, T., Wu, J., and Guan, K. L. (2002). TSC2 is phosphorylated and inhibited by Akt and suppresses mTOR signalling. Nat. Cell Biol. 4, 648-657. doi: 10.1038/ncb839

Inoki, K., Zhu, T., and Guan, K. L. (2003). TSC2 mediates cellular energy response to control cell growth and survival. Cell 115, 577-590. doi: 10.1016/S0092-8674(03)00929-2

Inukai, Y., Nonaka, T., Arai, T., Yoshida, M., Hashizume, Y., Beach, T. G., et al. (2008). Abnormal phosphorylation of Ser409/410 of TDP-43 in FTLD-U and ALS. FEBS Lett. 582, 2899-2904. doi: 10.1016/j.febslet.2008.07.027

Itakura, E., and Mizushima, N. (2010). Characterization of autophagosome formation site by a hierarchical analysis of mammalian Atg proteins. Autophagy 6, 764-776. doi: 10.4161/auto.6.6.12709 
Iwata, J., Ezaki, J., Komatsu, M., Yokota, S., Ueno, T., Tanida, I., et al. (2006). Excess peroxisomes are degraded by autophagic machinery in mammals. J. Biol. Chem. 281, 4035-4041. doi: 10.1074/jbc.M512283200

Jinwal, U. K., Abisambra, J. F., Zhang, J., Dharia, S., O’Leary, J. C., Patel, T., et al. (2012). Cdc37/Hsp90 protein complex disruption triggers an autophagic clearance cascade for TDP-43 protein. J. Biol. Chem. 287, 24814-24820. doi: $10.1074 /$ jbc.M112.367268

Johnson, B. S., McCaffery, J. M., Lindquist, S., and Gitler, A. D. (2008). A yeast TDP-43 proteinopathy model: Exploring the molecular determinants of TDP-43 aggregation and cellular toxicity. Proc. Natl. Acad. Sci. U.S.A. 105, 6439-6444. doi: 10.1073/pnas.0802082105

Johnson, B. S., Snead, D., Lee, J. J., Mccaffery, J. M., Shorter, J., and Gitler, A. D. (2009). TDP-43 Is Intrinsically aggregation-prone, and amyotrophic lateral sclerosis-linked mutations accelerate aggregation and increase toxicity. J. Biol. Chem. 284, 20329-20339. doi: 10.1074/jbc.M109.010264

Johnson, I. P. (2015). Age-related neurodegenerative disease research needs aging models. Front. Aging Neurosci. 7:168. doi: 10.3389/fnagi.2015.00168

Kanki, T. (2010). Nix, a receptor protein for mitophagy in mammals. Autophagy 6 , 433-435. doi: 10.4161/auto.6.3.11420

Katahira, J., Miki, T., Takano, K., Maruhashi, M., Uchikawa, M., Tachibana, T., et al. (2008). Nuclear RNA export factor 7 is localized in processing bodies and neuronal RNA granules through interactions with shuttling hnRNPs. Nucleic Acids Res. 36, 616-628. doi: 10.1093/nar/gkm556

Katsuragi, Y., Ichimura, Y., and Komatsu, M. (2015). p62/SQSTM1 functions as a signaling hub and an autophagy adaptor. FEBS J. 282, 4672-4678. doi: $10.1111 /$ febs. 13540

Kim, J., Kundu, M., Viollet, B., and Guan, K. L. (2011). AMPK and mTOR regulate autophagy through direct phosphorylation of Ulk1. Nat. Cell Biol. 13, 132-141. doi: $10.1038 / \mathrm{ncb} 2152$

King, A., Maekawa, S., Bodi, I., Troakes, C., and Al-Sarraj, S. (2011). Ubiquitinated, p62 immunopositive cerebellar cortical neuronal inclusions are evident across the spectrum of TDP-43 proteinopathies but are only rarely additionally immunopositive for phosphorylation-dependent TDP-43. Neuropathology 31, 239-249. doi: 10.1111/j.1440-1789.2010.01171.x

Klionsky, D. J., Abdelmohsen, K., Abe, A., Abedin, M. J., Abeliovich, H., Acevedo Arozena, A., et al. (2016). Guidelines for the use and interpretation of assays for monitoring autophagy (3rd edition). Autophagy 12, 1-222. doi: 10.1080/15548627.2015.1100356

Komatsu, M., Waguri, S., Chiba, T., Murata, S., Iwata, J., Tanida, I., et al. (2006). Loss of autophagy in the central nervous system causes neurodegeneration in mice. Nature 441, 880-884. doi: 10.1038/nature04723

Krasniak, C. S., and Ahmad, S. T. (2016). The role of CHMP2BIntron5 in autophagy and frontotemporal dementia. Brain Res. 1649, 151-157. doi: 10.1016/j.brainres.2016.02.051

Kuo, P.-H., Doudeva, L. G., Wang, Y.-T., Shen, C. -K. J., and Yuan, H. S. (2009). Structural insights into TDP-43 in nucleic-acid binding and domain interactions. Nucleic Acids Res. 37, 1799-1808. doi: 10.1093/nar/gkp013

Kwon, I., Xiang, S., Kato, M., Wu, L., Theodoropoulos, P., Wang, T., et al. (2014). Poly-dipeptides encoded by the C9orf72 repeats bind nucleoli, impede RNA biogenesis, and kill cells. Science 345, 1139-1145. doi: 10.1126/science.1254917

Lamb, C. A., Yoshimori, T., and Tooze, S. A. (2013). The autophagosome: origins unknown, biogenesis complex. Nat. Rev. Mol. Cell Biol. 14, 759-774. doi: $10.1038 / \mathrm{nrm} 3696$

Langellotti, S., Romano, V., Romano, G., Klima, R., Feiguin, F., Cragnaz, L., et al. (2016). A novel Drosophila model of TDP-43 proteinopathies: N-terminal sequences combined with the $\mathrm{Q} / \mathrm{N}$ domain induce protein functional loss and locomotion defects. Dis. Model. Mech. 9, 659-669. doi: 10.1242/dmm.023382

Lattante, S., Ciura, S., Rouleau, G. A., and Kabashi, E. (2015). Defining the genetic connection linking amyotrophic lateral sclerosis (ALS) with frontotemporal dementia (FTD). Trends Genet. 31, 263-273. doi: 10.1016/j.tig.2015.03.005

Lattante, S., Rouleau, G. A., and Kabashi, E. (2013). TARDBP and FUS mutations associated with amyotrophic lateral sclerosis: summary and update. Hum. Mutat. 34, 812-826. doi: 10.1002/humu.22319

Le Ber, I., Camuzat, A., Guerreiro, R., Bouya-Ahmed, K., Bras, J., Nicolas, G., et al. (2013). SQSTM1 mutations in French patients with frontotemporal dementia or frontotemporal dementia with amyotrophic lateral sclerosis. JAMA Neurol. 70, 1403-1410. doi: 10.1001/jamaneurol.2013.3849
Lee, E. B., Lee, V. M.-Y., and Trojanowski, J. Q. (2012). Gains or losses: molecular mechanisms of TDP43-mediated neurodegeneration. Nat. Rev. Neurosci. 13, 38-50. doi: 10.1038/nrn3121

Lee, J. A. (2012). Neuronal autophagy: a housekeeper or a fighter in neuronal cell survival? Exp. Neurobiol. 21, 1-8. doi: 10.5607/en.2012.21.1.1

Lee, J. A., Liu, L., and Gao, F. B. (2009). Autophagy defects contribute to neurodegeneration induced by dysfunctional ESCRT-III. Autophagy 5, 1070-1072. doi: 10.4161/auto.5.7.9823

Lee, S., Sato, Y., and Nixon, R. A. (2011). Lysosomal proteolysis inhibition selectively disrupts axonal transport of degradative organelles and causes an Alzheimer's-like axonal dystrophy. J. Neurosci. 31, 7817-7830. doi: 10.1523/JNEUROSCI.6412-10.2011

Levine, T. P., Daniels, R. D., Gatta, A. T., Wong, L. H., and Hayes, M. J. (2013). The product of C9orf72, a gene strongly implicated in neurodegeneration, is structurally related to DENN Rab-GEFs. Bioinformatics 29, 499-503. doi: 10.1093/bioinformatics/bts725

Lokk, J., and Delbari, A. (2012). Clinical aspects of palliative care in advanced Parkinson's disease. BMC Palliat. Care 11:20. doi: 10.1186/1472-684X-11-20

Mackenzie, I., Rademakers, R., and Neumann, M. (2010). TDP-43 and FUS in amyotrophic lateral sclerosis and frontotemporal dementia. Lancet Neurol. 9, 995-1007. doi: 10.1016/S1474-4422(10)70195-2

Maekawa, S., Leigh, P. N., King, A., Jones, E., Steele, J. C., Bodi, I., et al. (2009). TDP-43 is consistently co-localized with ubiquitinated inclusions in sporadic and Guam amyotrophic lateral sclerosis but not in familial amyotrophic lateral sclerosis with and without SOD1 mutations. Neuropathology 29, 672-683. doi: 10.1111/j.1440-1789.2009.01029.x

Maiuri, M. C., Criollo, A., Tasdemir, E., Vicencio, J. M., Tajeddine, N., Hickman, J. A., et al. (2007a). BH3-only proteins and BH3 mimetics induce autophagy by competitively disrupting the interaction between Beclin 1 and $\mathrm{Bcl}-2 / \mathrm{Bcl}-\mathrm{X}(\mathrm{L})$. Autophagy 3, 374-376. doi: 10.4161/auto.4237

Maiuri, M. C., Le Toumelin, G., Criollo, A., Rain, J. C., Gautier, F., Juin, P., et al. (2007b). Functional and physical interaction between Bcl-X(L) and a BH3-like domain in Beclin-1. EMBO J. 26, 2527-2539. doi: 10.1038/sj.emboj.7601689

Martinez-Vicente, M., Talloczy, Z., Wong, E., Tang, G., Koga, H., Kaushik, S., et al. (2010). Cargo recognition failure is responsible for inefficient autophagy in Huntington's disease. Nat. Neurosci. 13, 567-576. doi: 10.1038/nn.2528

Maruyama, H., Morino, H., Ito, H., Izumi, Y., Kato, H., Watanabe, Y., et al. (2010). Mutations of optineurin in amyotrophic lateral sclerosis. Nature 465, 223-226. doi: $10.1038 /$ nature 08971

Matsumoto, G., Shimogori, T., Hattori, N., and Nukina, N. (2015). TBK1 controls autophagosomal engulfment of polyubiquitinated mitochondria through p62/SQSTM1 phosphorylation. Hum. Mol. Genet. 24, 4429-4442. doi: $10.1093 / \mathrm{hmg} / \mathrm{ddv} 179$

Mazzulli, J. R., Xu, Y. H., Sun, Y., Knight, A. L., McLean, P. J., Caldwell, G. A., et al. (2011). Gaucher disease glucocerebrosidase and alpha-synuclein form a bidirectional pathogenic loop in synucleinopathies. Cell 146, 37-52. doi: 10.1016/j.cell.2011.06.001

Meijer, A. J., Lorin, S., Blommaart, E. F., and Codogno, P. (2015). Regulation of autophagy by amino acids and MTOR-dependent signal transduction. Amino Acids 47, 2037-2063. doi: 10.1007/s00726-014-1765-4

Menzies, F. M., Fleming, A., and Rubinsztein, D. C. (2015). Compromised autophagy and neurodegenerative diseases. Nat. Rev. Neurosci. 16, 345-357. doi: 10.1038/nrn3961

Mizumura, K., Choi, A. M., and Ryter, S. W. (2014). Emerging role of selective autophagy in human diseases. Front. Pharmacol. 5:244. doi: 10.3389/fphar.2014.00244

Mizuno, Y., Amari, M., Takatama, M., Aizawa, H., Mihara, B., and Okamoto, K. (2006). Immunoreactivities of p62, an ubiqutin-binding protein, in the spinal anterior horn cells of patients with amyotrophic lateral sclerosis. J. Neurol. Sci. 249, 13-18. doi: 10.1016/j.jns.2006.05.060

Moreau, K., Renna, M., and Rubinsztein, D. C. (2013). Connections between SNAREs and autophagy. Trends Biochem. Sci. 38, 57-63. doi: 10.1016/j.tibs.2012.11.004

Moreau, K., and Rubinsztein, D. C. (2012). The plasma membrane as a control center for autophagy. Autophagy 8, 861-863. doi: 10.4161/auto.20060

Murphy, K. E., Gysbers, A. M., Abbott, S. K., Tayebi, N., Kim, W. S., Sidransky, E., et al. (2014). Reduced glucocerebrosidase is associated with 
increased alpha-synuclein in sporadic Parkinson's disease. Brain 137, 834-848. doi: 10.1093/brain/awt367

Napolitano, G., and Ballabio, A. (2016). TFEB at a glance. J. Cell Sci. 129, 2475-2481. doi: 10.1242/jcs. 146365

Narendra, D., Tanaka, A., Suen, D. F., and Youle, R. J. (2008). Parkin is recruited selectively to impaired mitochondria and promotes their autophagy. J. Cell Biol. 183, 795-803. doi: 10.1083/jcb.200809125

Natale, G., Lenzi, P., Lazzeri, G., Falleni, A., Biagioni, F., Ryskalin, L., et al. (2015). Compartment-dependent mitochondrial alterations in experimental ALS, the effects of mitophagy and mitochondriogenesis. Front. Cell. Neurosci. 9:434. doi: 10.3389/fncel.2015.00434

Neumann, M., Sampathu, D. M., Kwong, L. K., Truax, A. C., Micsenyi, M. C., Chou, T. T., et al. (2006). Ubiquitinated TDP-43 in frontotemporal lobar degeneration and amyotrophic lateral sclerosis. Science 314, 130-133. doi: 10.1126/science.1134108

Nijholt, D. A., De Kimpe, L., Elfrink, H. L., Hoozemans, J. J., and Scheper, W. (2011). Removing protein aggregates: the role of proteolysis in neurodegeneration. Curr. Med. Chem. 18, 2459-2476. doi: $10.2174 / 092986711795843236$

Oakes, J. A., Davies, M. C., and Collins, M. O. (2017). TBK1: a new player in ALS linking autophagy and neuroinflammation. Mol. Brain 10, 5. doi: 10.1186/s13041-017-0287-x

Onesto, E., Colombrita, C., Gumina, V., Borghi, M. O., Dusi, S., Doretti, A., et al. (2016). Gene-specific mitochondria dysfunctions in human TARDBP and C9ORF72 fibroblasts. Acta Neuropathol. Commun. 4, 47. doi: 10.1186/s40478-016-0316-5

Orlova, K. A., and Crino, P. B. (2010). The tuberous sclerosis complex. Ann. N. Y. Acad. Sci. 1184, 87-105. doi: 10.1111/j.1749-6632.2009. 05117.x

Otomo, C., Metlagel, Z., Takaesu, G., and Otomo, T. (2013). Structure of the human ATG12 ATG5 conjugate required for LC3 lipidation in autophagy. Nat. Struct. Mol. Biol. 20, 59-66. doi: 10.1038/nsmb.2431

Ou, S. H., Wu, F., Harrich, D., García-Martínez, L. F., and Gaynor, R. B. (1995). Cloning and characterization of a novel cellular protein, TDP-43, that binds to human immunodeficiency virus type 1 TAR DNA sequence motifs. J. Virol. 69, 3584-3596.

Parkinson, N., Ince, P. G., Smith, M. O., Highley, R., Skibinski, G., Andersen, P. M., et al. (2006). ALS phenotypes with mutations in CHMP2B (charged multivesicular body protein 2B). Neurology 67, 1074-1077. doi: 10.1212/01.wnl.0000231510.89311.8b

Pasquali, L., Longone, P., Isidoro, C., Ruggieri, S., Paparelli, A., and Fornai, F. (2009). Autophagy, lithium, and amyotrophic lateral sclerosis. Muscle Nerve 40, 173-194. doi: 10.1002/mus.21423

Patergnani, S., Missiroli, S., Marchi, S., and Giorgi, C. (2015). MitochondriaAssociated Endoplasmic reticulum membranes microenvironment: targeting autophagic and apoptotic pathways in cancer therapy. Front. Oncol. 5:173. doi: 10.3389/fonc.2015.00173

Pickford, F., Masliah, E., Britschgi, M., Lucin, K., Narasimhan, R., Jaeger, P. A., et al. (2008). The autophagy-related protein beclin 1 shows reduced expression in early Alzheimer disease and regulates amyloid beta accumulation in mice. $J$. Clin. Invest. 118, 2190-2199. doi: 10.1172/JCI33585

Polson, H. E., de Lartigue, J., Rigden, D. J., Reedijk, M., Urbe, S., Clague, M. J., et al. (2010). Mammalian Atg18 (WIPI2) localizes to omegasome-anchored phagophores and positively regulates LC3 lipidation. Autophagy 6, 506-522. doi: 10.4161/auto.6.4.11863

Potter, C. J., Pedraza, L. G., and Xu, T. (2002). Akt regulates growth by directly phosphorylating Tsc2. Nat. Cell Biol. 4, 658-665. doi: 10.1038/ncb840

Proikas-Cezanne, T., Waddell, S., Gaugel, A., Frickey, T., Lupas, A., and Nordheim, A. (2004). WIPI-1 $\alpha$ (WIPI49), a member of the novel 7-bladed WIPI protein family, is aberrantly expressed in human cancer and is linked to starvation-induced autophagy. Oncogene 23, 9314-9325. doi: 10.1038/sj.onc.12 08331

Prpar Mihevc, S., Baralle, M., Buratti, E., and Rogelj, B. (2016). TDP-43 aggregation mirrors TDP-43 knockdown, affecting the expression levels of a common set of proteins. Sci. Rep. 6, 33996. doi: 10.1038/srep33996

Ratti, A., and Buratti, E. (2016). Physiological functions and pathobiology of TDP-43 and FUS/TLS proteins. J. Neurochem. 138(Suppl. 1), 95-111. doi: $10.1111 /$ jnc. 13625
Ravikumar, B., Acevedo-Arozena, A., Imarisio, S., Berger, Z., Vacher, C., O’Kane, C. J., et al. (2005). Dynein mutations impair autophagic clearance of aggregateprone proteins. Nat. Genet. 37, 771-776. doi: 10.1038/ng1591

Ravikumar, B., Moreau, K., Jahreiss, L., Puri, C., and Rubinsztein, D. C. (2010). Plasma membrane contributes to the formation of pre-autophagosomal structures. Nat. Cell Biol. 12, 747-757. doi: 10.1038/ncb2078

Ravikumar, B., Vacher, C., Berger, Z., Davies, J. E., Luo, S., Oroz, L. G., et al. (2004). Inhibition of mTOR induces autophagy and reduces toxicity of polyglutamine expansions in fly and mouse models of Huntington disease. Nat. Genet. 36, 585-595. doi: 10.1038/ng1362

Reeve, A., Simcox, E., and Turnbull, D. (2014). Ageing and Parkinson's disease: why is advancing age the biggest risk factor? Ageing Res. Rev. 14, 19-30. doi: 10.1016/j.arr.2014.01.004

Renton, A. E., Majounie, E., Waite, A., Simon-Sanchez, J., Rollinson, S., Gibbs, J. R., et al. (2011). A hexanucleotide repeat expansion in C9ORF72 is the cause of chromosome 9p21-linked ALS-FTD. Neuron 72, 257-268. doi: 10.1016/j.neuron.2011.09.010

Richter, B., Sliter, D. A., Herhaus, L., Stolz, A., Wang, C., Beli, P., et al. (2016). Phosphorylation of OPTN by TBK1 enhances its binding to Ub chains and promotes selective autophagy of damaged mitochondria. Proc. Natl. Acad. Sci. U.S.A. 113, 4039-4044. doi: 10.1073/pnas.1523926113

Rohrer, J. D., Isaacs, A. M., Mizielinska, S., Mead, S., Lashley, T., Wray, S., et al. (2015). C9orf72 expansions in frontotemporal dementia and amyotrophic lateral sclerosis. Lancet Neurol. 14, 291-301. doi: 10.1016/S1474-4422(14)70233-9

Romano, V., Quadri, Z., Baralle, F. E., and Buratti, E. (2015). The structural integrity of TDP-43 N-terminus is required for efficient aggregate entrapment and consequent loss of protein function. Prion 9, 1-9. doi: 10.1080/19336896.2015.1011885

Russell, R. C., Yuan, H. X., and Guan, K. L. (2014). Autophagy regulation by nutrient signaling. Cell Res. 24, 42-57. doi: 10.1038/cr.2013.166

Sarkar, S., Floto, R. A., Berger, Z., Imarisio, S., Cordenier, A., Pasco, M., et al. (2005). Lithium induces autophagy by inhibiting inositol monophosphatase. J. Cell Biol. 170, 1101-1111. doi: 10.1083/jcb.200504035

Sarkar, S., Ravikumar, B., and Rubinsztein, D. C. (2009). Autophagic clearance of aggregate-prone proteins associated with neurodegeneration. Meth. Enzymol. 453, 83-110. doi: 10.1016/S0076-6879(08)04005-6

Sasaki, S. (2011). Autophagy in spinal cord motor neurons in sporadic amyotrophic lateral sclerosis. J. Neuropathol. Exp. Neurol. 70, 349-359. doi: 10.1097/NEN.0b013e3182160690

Scotter, E. L., Vance, C., Nishimura, A. L., Lee, Y. B., Chen, H. J., Urwin, H., et al. (2014). Differential roles of the ubiquitin proteasome system and autophagy in the clearance of soluble and aggregated TDP-43 species. J. Cell Sci. 127, 1263-1278. doi: 10.1242/jcs. 140087

Sellier, C., Campanari, M. L., Julie Corbier, C., Gaucherot, A., Kolb-Cheynel, I., Oulad-Abdelghani, M., et al. (2016). Loss of C9ORF72 impairs autophagy and synergizes with polyQ Ataxin-2 to induce motor neuron dysfunction and cell death. EMBO J. 35, 1276-1297. doi: 10.15252/embj.201593350

Shen, W. C., Li, H. Y., Chen, G. C., Chern, Y., and Tu, P. H. (2015). Mutations in the ubiquitin-binding domain of OPTN/optineurin interfere with autophagy-mediated degradation of misfolded proteins by a dominantnegative mechanism. Autophagy 11, 685-700. doi: 10.4161/auto.36098

Shibata, M., Lu, T., Furuya, T., Degterev, A., Mizushima, N., Yoshimori, T., et al. (2006). Regulation of intracellular accumulation of mutant Huntingtin by Beclin 1. J. Biol. Chem. 281, 14474-14485. doi: 10.1074/jbc.M600364200

Shimizu, H., Toyoshima, Y., Shiga, A., Yokoseki, A., Arakawa, K., Sekine, Y., et al. (2013). Sporadic ALS with compound heterozygous mutations in the SQSTM1 gene. Acta Neuropathol. 126, 453-459. doi: 10.1007/s00401-013-1150-5

Simonsen, A., Birkeland, H. C., Gillooly, D. J., Mizushima, N., Kuma, A., Yoshimori, T., et al. (2004). Alfy, a novel FYVE-domain-containing protein associated with protein granules and autophagic membranes. J. Cell Sci. 117, 4239-4251. doi: 10.1242/jcs.01287

Skibinski, G., Parkinson, N. J., Brown, J. M., Chakrabarti, L., Lloyd, S. L., Hummerich, H., et al. (2005). Mutations in the endosomal ESCRTIII-complex subunit CHMP2B in frontotemporal dementia. Nat. Genet. 37, 806-808. doi: $10.1038 /$ ng1609

Spinosa, M. R., Progida, C., De Luca, A., Colucci, A. M., Alifano, P., and Bucci, C. (2008). Functional characterization of Rab7 mutant proteins associated 
with Charcot-Marie-Tooth type 2B disease. J. Neurosci. 28, 1640-1648. doi: 10.1523/JNEUROSCI.3677-07.2008

Sullivan, P. M., Zhou, X., Robins, A. M., Paushter, D. H., Kim, D., Smolka, M. B., et al. (2016). The ALS/FTLD associated protein C9orf72 associates with SMCR8 and WDR41 to regulate the autophagy-lysosome pathway. Acta Neuropathol. Commun. 4:51. doi: 10.1186/s40478-016-0324-5

Tanida, I., Sou, Y. S., Ezaki, J., Minematsu-Ikeguchi, N., Ueno, T., and Kominami, E. (2004). HsAtg4B/HsApg4B/autophagin-1 cleaves the carboxyl termini of three human Atg8 homologues and delipidates microtubule-associated protein light chain 3- and GABAA receptor-associated protein-phospholipid conjugates. J. Biol. Chem. 279, 36268-36276. doi: 10.1074/jbc.M401461200

Tasdemir, E., Maiuri, M. C., Tajeddine, N., Vitale, I., Criollo, A., Vicencio, J. M., et al. (2007). Cell cycle-dependent induction of autophagy, mitophagy and reticulophagy. Cell Cycle 6, 2263-2267. doi: 10.4161/cc.6.18.4681

Teyssou, E., Takeda, T., Lebon, V., Boillee, S., Doukoure, B., Bataillon, G., et al. (2013). Mutations in SQSTM1 encoding p62 in amyotrophic lateral sclerosis: genetics and neuropathology. Acta Neuropathol. 125, 511-522. doi: 10.1007/s00401-013-1090-0

Todd, T. W., and Petrucelli, L. (2016). Insights into the pathogenic mechanisms of Chromosome 9 open reading frame 72 (C9orf72) repeat expansions. J. Neurochem. 138(Suppl. 1), 145-162. doi: 10.1111/jnc.13623

Troakes, C., Maekawa, S., Wijesekera, L., Rogelj, B., Siklos, L., Bell, C., et al. (2012). An MND/ALS phenotype associated with C9orf72 repeat expansion: abundant p62-positive, TDP-43-negative inclusions in cerebral cortex, hippocampus and cerebellum but without associated cognitive decline. Neuropathology 32, 505-514. doi: 10.1111/j.1440-1789.2011.01286.x

Urushitani, M., Sato, T., Bamba, H., Hisa, Y., and Tooyama, I. (2010). Synergistic effect between proteasome and autophagosome in the clearance of polyubiquitinated TDP-43. J. Neurosci. Res. 88, 784-797. doi: 10.1002/jnr.22243

Uusi-Rauva, K., Kyttala, A., van der Kant, R., Vesa, J., Tanhuanpaa, K., Neefjes, J., et al. (2012). Neuronal ceroid lipofuscinosis protein CLN3 interacts with motor proteins and modifies location of late endosomal compartments. Cell. Mol. Life Sci. 69, 2075-2089. doi: 10.1007/s00018-011-0913-1

Walczak, M., and Martens, S. (2013). Dissecting the role of the Atg12-Atg5Atg16 complex during autophagosome formation. Autophagy 9, 424-425. doi: 10.4161/auto.22931

Wang, I. F., Guo, B. S., Liu, Y. C., Wu, C. C., Yang, C. H., Tsai, K. J., et al. (2012). Autophagy activators rescue and alleviate pathogenesis of a mouse model with proteinopathies of the TAR DNA-binding protein 43. Proc. Natl. Acad. Sci. U.S.A. 109, 15024-15029. doi: 10.1073/pnas.1206362109

Wang, X., Fan, H., Ying, Z., Li, B., Wang, H., and Wang, G. (2010). Degradation of TDP-43 and its pathogenic form by autophagy and the ubiquitin-proteasome system. Neurosci. Lett. 469, 112-116. doi: 10.1016/j.neulet.2009.11.055

Wang, X., Zhou, S., Ding, X., Ma, M., Zhang, J., Zhou, Y., et al. (2015). Activation of ER stress and autophagy induced by TDP-43 A315T as pathogenic mechanism and the corresponding histological changes in skin as potential biomarker for ALS with the mutation. Int. J. Biol. Sci. 11, 1140-1149. doi: 10.7150/ijbs. 12657

Webster, C. P., Smith, E. F., Bauer, C. S., Moller, A., Hautbergue, G. M., Ferraiuolo, L., et al. (2016). The C9orf72 protein interacts with Rabla and the ULK1 complex to regulate initiation of autophagy. EMBO J. 35, 1656-1676. doi: $10.15252 / \mathrm{embj} .201694401$
Winslow, A. R., Chen, C. W., Corrochano, S., Acevedo-Arozena, A., Gordon, D. E., Peden, A. A., et al. (2010). alpha-Synuclein impairs macroautophagy: implications for Parkinson's disease. J. Cell Biol. 190, 1023-1037. doi: $10.1083 /$ jcb.201003122

Wong, P. M., Puente, C., Ganley, I. G., and Jiang, X. (2013). The ULK1 complex: sensing nutrient signals for autophagy activation. Autophagy 9, 124-137. doi: 10.4161 /auto. 23323

Wong, Y. C., and Holzbaur, E. L. (2014). Optineurin is an autophagy receptor for damaged mitochondria in parkin-mediated mitophagy that is disrupted by an ALS-linked mutation. Proc. Natl. Acad. Sci. U.S.A. 111, E4439-E4448. doi: 10.1073/pnas.1405752111

Xia, Q., Wang, H., Hao, Z., Fu, C., Hu, Q., Gao, F., et al. (2016). TDP-43 loss of function increases TFEB activity and blocks autophagosome-lysosome fusion. EMBO J. 35, 121-142. doi: 10.15252/embj.201591998

Yang, M., Liang, C., Swaminathan, K., Herrlinger, S., Lai, F., Shiekhattar, R., et al. (2016). A C9ORF72/SMCR8-containing complex regulates ULK1 and plays a dual role in autophagy. Sci. Adv. 2:e1601167. doi: 10.1126/sciadv.1601167

Ying, Z., Xia, Q., Hao, Z., Xu, D., Wang, M., Wang, H., et al. (2016). TARDBP/TDP-43 regulates autophagy in both MTORC1-dependent and MTORC1-independent manners. Autophagy 12, 707-708. doi: 10.1080/15548627.2016.1151596

Yla-Anttila, P., Vihinen, H., Jokitalo, E., and Eskelinen, E. L. (2009). 3D tomography reveals connections between the phagophore and endoplasmic reticulum. Autophagy 5, 1180-1185. doi: 10.4161/auto.5.8.10274

Zerial, M., and McBride, H. (2001). Rab proteins as membrane organizers. Nat. Rev. Mol. Cell Biol. 2, 107-117. doi: 10.1038/35052055

Zhang, D., Iyer, L. M., He, F., and Aravind, L. (2012). Discovery of novel DENN proteins: implications for the evolution of eukaryotic intracellular membrane structures and human disease. Front. Genet. 3:283. doi: 10.3389/fgene.2012.00283

Zhang, Y.-J., Xu, Y.-F., Cook, C., Gendron, T. F., Roettges, P., Petrucelli, L., et al. (2009). Aberrant cleavage of TDP-43 enhances aggregation and cellular toxicity. Proc. Natl. Acad. Sci. U.S.A. 106, 7607-7612. doi: 10.1073/pnas.0900688106

Zhong, Y., Wang, Q. J., Li, X., Yan, Y., Backer, J. M., Chait, B. T., et al. (2009). Distinct regulation of autophagic activity by Atg14L and Rubicon associated with Beclin 1-phosphatidylinositol-3-kinase complex. Nat. Cell Biol. 11, 468-476. doi: 10.1038/ncb1854

Zu, T., Liu, Y., Banez-Coronel, M., Reid, T., Pletnikova, O., Lewis, J., et al. (2013). RAN proteins and RNA foci from antisense transcripts in C9ORF72 ALS and frontotemporal dementia. Proc. Natl. Acad. Sci. U.S.A. 110, E4968-E4977. doi: 10.1073/pnas. 1315438110

Conflict of Interest Statement: The authors declare that the research was conducted in the absence of any commercial or financial relationships that could be construed as a potential conflict of interest.

Copyright (C) 2017 Budini, Buratti, Morselli and Criollo. This is an open-access article distributed under the terms of the Creative Commons Attribution License (CC BY). The use, distribution or reproduction in other forums is permitted, provided the original author(s) or licensor are credited and that the original publication in this journal is cited, in accordance with accepted academic practice. No use, distribution or reproduction is permitted which does not comply with these terms. 